\title{
Development of a Web-Based Toolbox to Support Quantitative In- Vitro-to-In-Vivo Extrapolations (QIVIVE) within Nonanimal Testing Strategies
}

Ans Punt,* Nicole Pinckaers, Ad Peijnenburg, and Jochem Louisse

Cite This: Chem. Res. Toxicol. 2021, 34, 460-472

Read Online

Supporting Information

ABSTRACT: The goal of the present study was to develop an online web-based toolbox that contains generic physiologically based kinetic (PBK) models for rats and humans, including underlying calculation tools to predict plasma protein binding and tissue:plasma distribution, to be used for quantitative in-vitro-to-invivo extrapolations (QIVIVE). The PBK models within the toolbox allow first estimations of internal plasma and tissue concentrations of chemicals to be made, based on the $\log \mathrm{P}$ and $\mathrm{p} K_{\mathrm{a}}$ of the chemicals and values for intestinal uptake and intrinsic hepatic clearance. As a case study, the toolbox was used to predict oral equivalent doses of in vitro ToxCast bioactivity data for the food additives methylparaben, propyl gallate, octyl gallate, and dodecyl gallate. These oral equivalent doses were subsequently compared with human exposure estimates, as a low tier assessment allowing prioritization for further assessment. The results revealed that daily intake levels of especially propyl gallate can lead to internal plasma concentrations that are close to in vitro biological effect concentrations, particularly with respect to the inhibition of human thyroid peroxidase (TPO). Estrogenic effects were not considered likely to be induced by the food additives, as daily exposure levels of the different compounds remained 2 orders of magnitude below the oral equivalent doses for in vitro estrogen receptor activation. Overall, the results of the study show how the toolbox, which is freely accessible through www.qivivetools.wur.nl, can be used to obtain initial internal dose estimates of chemicals and to prioritize chemicals for further assessment, based on the comparison of oral equivalent doses of in vitro biological activity data with human exposure levels.

\section{INTRODUCTION}

An important aspect within next-generation nonanimal toxicity testing strategies is the extrapolation of in vitro effect concentrations into (human) dose-response or potency information, also called quantitative in-vitro-to-in-vivo extrapolation (QIVIVE). ${ }^{1-4}$ For QIVIVE, insight in dose-dependent internal concentrations of a chemical (e.g., in plasma or in a tissue) is required to infer the dose level that is needed to reach the internal concentration that causes an effect in an in vitro assay, also known as an oral equivalent dose. Physiologically based kinetic (PBK) modeling plays a crucial role to provide such insights into dose-dependent internal concentrations required to derive oral equivalent doses.

In nonanimal toxicity testing strategies, PBK models are needed that are developed based on nonanimal approaches, so that the results solely depend on in vitro and in silico derived chemical input parameters. These input parameters need to be integrated in (generic) PBK models, allowing prediction of dose-dependent internal concentrations required to derive oral equivalent doses. Development of PBK models is not straightforward, requiring access to PBK-modeling software and a basic understanding of and experience with PBK modeling and the generation of in vitro and in silico input parameters. When lacking these prerequisites, the application of QIVIVE approaches by in vitro toxicologists without PBKmodeling experience may be hampered. In order to promote the use of PBK modeling and QIVIVE, the goal of the present study is to build a user-friendly web-based toolbox that facilitates QIVIVE, also providing a tool for nonexperienced PBK modelers.

The toolbox contains a collection of calculators for the estimation of chemical-specific in silico derived input parameters for PBK models, such as the fraction unbound in plasma (fup) and tissue:plasma partition coefficients. For these calculators, information on the $\log \mathrm{P}$ and $\mathrm{p} K_{\mathrm{a}}$ of the chemical is required. Values on fup and/or tissue:plasma partition coefficients can also be directly inserted in the tool by the

Special Issue: Computational Toxicology

Received: July 30, 2020

Published: December 31, 2020

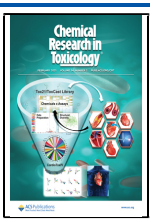


user, e.g., when experimental data are available. The user also needs to add information on the velocity of intestinal uptake and intrinsic hepatic clearance obtained by either hepatocytes or liver tissue fractions (microsomes or S9). The calculated or inserted chemical-specific kinetic information is automatically integrated in the PBK-model code, allowing quick generation of estimations of internal chemical concentrations (e.g., in plasma and in organs) at exposure scenarios as determined by the user. This indicates that in order to make estimations of dose-dependent internal concentrations, only limited information is required, being the $\log \mathrm{P}$ and $\mathrm{p} K_{\mathrm{a}}$ of the chemical and a value for intestinal uptake and intrinsic (in vitro) hepatic clearance. The obtained predicted dose-dependent internal concentrations can be linked to in vitro effect (toxicity) data of the chemical to estimate at what dose levels such effect concentrations are reached, i.e., to derive an oral equivalent dose.

To provide more information on the use of the developed web-based toolbox for QIVIVE, it was applied in the present study to obtain oral equivalent doses of in vitro ToxCast bioactivity data for the chemicals methylparaben, propyl gallate, octyl gallate, and dodecyl gallate. These compounds (Figure 1) are structurally related food additives, with

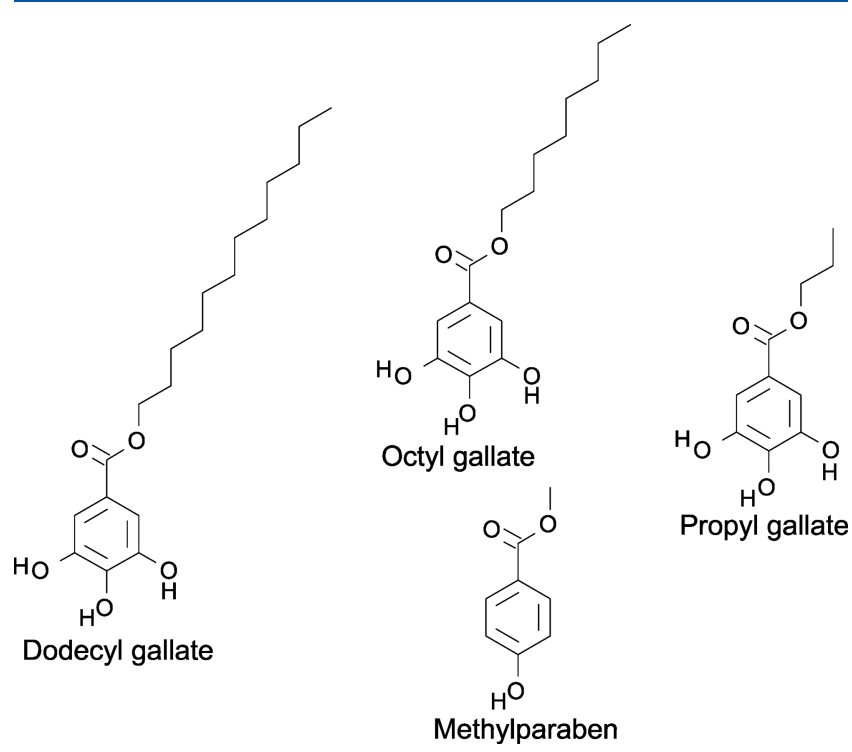

Figure 1. Molecular structures of methylparaben and the different gallates included in the present study.

methylparaben being used as a preservative and the different gallates being used as antioxidants. ${ }^{5,6}$ Parabens are known to be estrogenic in vitro as well as in the uterotrophic assay in vivo. $^{9-10}$ The estrogenic potency of methylparaben has been reported to be relatively low compared with those of longer chain parabens. ${ }^{11,12}$ Gallates like propyl gallate, octyl gallate, and dodecyl gallate also display estrogenic activity in vitro, though only at relatively high concentrations that are close to cytotoxic concentrations. ${ }^{13-15}$ Methylparaben is authorized for use in certain food products, such as for surface treatment of dried meat products. ${ }^{5}$ Also, propyl gallate is authorized to be used in food ${ }^{16}$ and is, for example, used in aqueous vitamin D supplements for babies and toddlers. The use of both dodecyl gallate and octyl gallate in food has recently been suspended, due to a lack of detailed reports on carcinogenicity and chronic toxicity studies. ${ }^{17-19}$ Also, the toxicity data available for propyl gallate are limited, as indicated by a recent evaluation by the Dutch National Institute for Public Health and the Environment (RIVM), pointing to the lack of an extended onegeneration reproductive toxicity study, which was deemed necessary to derive an acceptable daily intake (ADI) for babies younger than 16 weeks. $^{20}$

In the present study, we used the web-based toolbox to calculate oral equivalent doses for methylparaben, propyl gallate, octyl gallate, and dodecyl gallate based on in vitro effect concentrations as obtained in the ToxCast and Tox 21 programs. $^{21}$ By comparing these calculated oral equivalent doses with information on human exposure, the chemicals with most concern can be identified, allowing prioritization for further assessment. The ultimate goal of the toolbox is to make PBK modeling and QIVIVE widely accessible, including to scientists without PBK-modeling experience. Furthermore, apart from developing and running a human or rat PBK model for nonexperienced PBK modelers, the toolbox can serve as a repository of in silico tools to calculate chemicalspecific PBK-model parameter values, including tissue:plasma partition coefficients and fraction unbound in plasma.

\section{MATERIALS AND METHODS}

2.1. Model Code and Software. The QIVIVE toolbox is coded in $\mathrm{R}^{22}$ and $\mathrm{R}$ shiny (an R package for building interactive web apps), ${ }^{23}$ and contains a generic PBK model for rats and for humans. The differential equations of the models are solved with the deSolve package. ${ }^{24}$ The $\mathrm{R}$ codes of the rat and human PBK models underlying the online web application are provided in the Supporting Information 1. In addition, these codes can be found on https:// github.com/wfsrqivive/pbktool, providing also a platform to report issues and questions related to the toolbox. The human model is constructed on the basis of a published human model by Jones and Rowland-Yeo (Figure 2). ${ }^{25}$ For the rat model, the same model code is applied, replacing the human physiological parameters with parameters for the rat as obtained from Musther et al. ${ }^{26}$ The models simulate plasma concentrations in rats and humans based on chemical-specific parameters for intestinal uptake, distribution (i.e., partition coefficients, blood:plasma ratio, fraction unbound in plasma), hepatic clearance, and renal clearance (assumed to be the

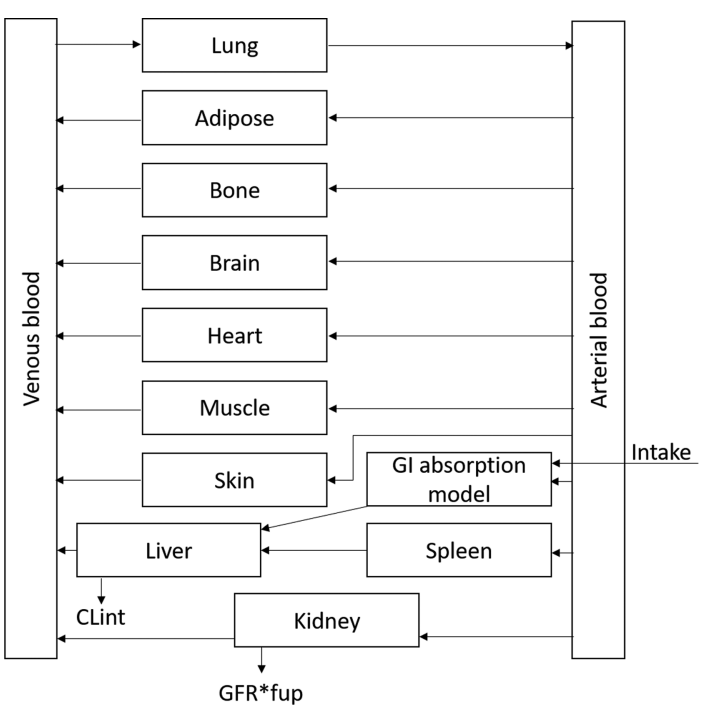

Figure 2. Structure of the PBK model that is integrated in the toolbox. CLint corresponds to the intrinsic hepatic clearance, GFR corresponds to the glomerular filtration rate, and fup corresponds to the fraction unbound in plasma. 
the glomerular filtration rate (values taken from Grandoni et al. ${ }^{27}$ ) multiplied by the free concentration, thereby neglecting processes of active secretion and tubular reabsorption). The developed toolbox can be accessed through www.qivivetools.wur.nl.

To run the PBK models for any chemical of interest, a few steps in the PBK workflow need to be followed, which is shortly described below. In the Supporting Information 2, the workflow is also shown with help of screenshots of the online tool. First, chemical-specific information $\left(\log \mathrm{P}, \mathrm{p} K_{\mathfrak{a}}\right.$ and molecular weight) needs to be inserted ("Physicochemical parameters" tab of PBK Workflow). Calculators available in the literature to predict plasma:tissue partition coefficients $^{28,29}$ and the fraction unbound in plasma ${ }^{30}$ based on the chemical's $\log \mathrm{P}$ and $\mathrm{p} K_{\mathrm{a}}$ as input are integrated in the toolbox. Users need to select the calculator they wish to use for the prediction of partition coefficients, which are automatically applied in the generic PBK model. Both provided methods are based on estimated partitioning between water, lipid, and protein fractions in plasma and tissues, requiring information on the lipophilicity (based on $\log \mathrm{P}$ ) and charge (based on $\mathrm{pK}_{\mathrm{a}}$ ) of the chemicals. The models have been developed based on these general principles as described in the underlying equations, which are not mathematically derived equations to best fit a training data set. Evaluation of these methods is based on data for pharmaceuticals, so it remains to be established how well these calculators apply for other chemicals. The calculator available in the tool to estimate the fraction unbound in plasma is based on a method of Lobell and Sivarajah ${ }^{30}$ that uses $\log \mathrm{P}$ and information on the charge as input to predict the fraction unbound for chemicals grouped in uncharged, negatively charged, positively charged with a $\log \mathrm{P} \leq 0.2$, positively charged with a $\log \mathrm{P}>0.2$, zwitterionic, or permanently positively charged (irrespective of $\mathrm{pH}$, containing quarternary nitrogen) groups. This information is automatically incorporated in the calculations carried out by the web-based tool based on the information the user added regarding the physicochemical characteristics in the first tab of the online tool. Currently, no adequate calculators for the estimation of the blood:plasma ratio are available, and only custom-derived values can be included in the webbased tool. If no information on the blood:plasma ratio is available, the blood:plasma ratio can be set to 1 as a default approach.

In the second tab of the web-based toolbox, in vitro intrinsic hepatic clearance data needs to be included for the species of interest ("Metabolism" tab of PBK Workflow). Scaling values are included in the toolbox for different in vitro systems $(\mathrm{S} 9$, microsomes, or primary hepatocytes $)^{26,31,32}$ to scale the inserted in vitro hepatic intrinsic clearance values to in vivo intrinsic hepatic clearance values, which are automatically integrated in the PBK model. The metabolism tab also contains calculators to predict the fraction unbound in the in vitro clearance incubations, which is integrated in the PBK model to correct the intrinsic clearance for nonspecific binding to the enzyme source. $^{33,34}$ The calculation method by Hallifax and Houston ${ }^{34}$ for the prediction of the fraction unbound in microsomal incubations is based on a regression analysis between $\log \mathrm{P} / \mathrm{D}$ and measured unbound fractions for a range of pharmaceuticals. Making use of the correlation between the fraction unbound in microsomal incubation and incubations with hepatocytes, Kilford et al. ${ }^{33}$ adjusted the equation of Hallifax and Houston ${ }^{34}$ to obtain an equation that allows the fraction unbound in hepatocyte incubations to be predicted. Within the toolbox, the required input for the calculation of the fraction unbound in the in vitro incubations is derived from the physicochemical properties tab. In addition, the concentration hepatocytes, microsomes, or $\mathrm{S} 9$ needs to be provided.

Regarding intestinal absorption, an absorption rate constant $\left(k_{\mathrm{a}}\right)$ needs to be added as input ("Intestinal uptake" tab of PBK Workflow). A $k_{\mathrm{a}}$ value can be estimated based on Papp values determined with Caco-2 transport studies, which are estimated based on a calculation using information on the chemical's topological polar surface area (TPSA) (see more details in Section 2.3). The oral uptake (using $k_{\mathrm{a}}$ as input) is described in the generic PBK-model code used in the web-based toolbox with a seven-compartment model according to the compartmental absorption and transit (CAT) model of $\mathrm{Yu}$ and Amidon. ${ }^{35}$ The first compartment represents the stomach, the last compartment represents the colon, and the other segments represent the small intestine. Absorption in this model only takes place from the small intestinal segments and not from the colon or stomach. The physiological data to describe the different intestinal segments and the transit times were obtained from Grandoni et al. ${ }^{27}$ When the oral uptake is relatively low, a chemical will reach the colon compartment and the fraction absorbed will be lower than 1 . The gastrointestinal model therefore automatically accounts for a fraction absorbed $(\mathrm{Fa})$ lower than 1 for chemicals with a slow uptake. The gastrointestinal model included in the generic PBK model used does not account for dissolution and assumes that the compound is completely dissolved in the matrix when dosed.

The PBK-model predictions are shown in the "PBK result" tab. In this tab, different dosing scenarios (dose, dose rate, and exposure duration) can be applied, and model results change accordingly. Model results can be exported to csv files for further use. Both total and free (unbound) concentrations in plasma and tissues can be displayed. By simulating the plasma concentrations at different dose levels, one can infer the dose that is needed to reach peak plasma concentrations that equal reported effect concentrations of the chemical, allowing derivation of oral equivalent doses.

In the present study, the toolbox is used to predict plasma concentrations of methyparaben, propyl gallate, octyl gallate, and dodecyl gallate and to extrapolate in vitro ToxCast bioactivity data into oral equivalent doses. To that end, in vitro experiments with human liver S9 (HLS9) and rat liver S9 (RLS9) were performed to obtain input parameter values for intrinsic hepatic clearance (CLint) of these chemicals, whereas the remaining input parameters (fup, tissue:plasma partition coefficients, nonspecific binding to $S 9$, and $k_{\mathrm{a}}$ ) were estimated based on the physicochemical characteristics of the chemicals $\left(\log \mathrm{P}, \mathrm{p} K_{a}\right.$ and TPSA)

2.2. In Vitro Metabolism Study with Human and Rat Liver S9. The CLint values of methyparaben (Sigma-Aldrich, Steinheim, Germany), propyl gallate (European Pharmacopoeia Reference, Strasbourg, France), octyl gallate (European Pharmacopoeia Reference, Strasbourg, France), and dodecyl gallate (European Pharmacopoeia Reference, Strasbourg, France) were determined in incubations with pooled human liver S9 (Corning, Woburn, USA, lotnr 9021003) and pooled male Sprague-Dawley rat (from Corning, Woburn, USA, lotnr 9017001). For these experiments, stock solutions of the different compounds of $1 \mathrm{mM}$ were prepared in DMSO (Mallinckrodt Baker B.V., Deventer, The Netherlands) and further diluted to $100 \mu \mathrm{M}$ in $100 \mathrm{mM}$ potassium phosphate buffer (Sigma-Aldrich, Steinheim, Germany) with $5 \mathrm{mM}$ magnesium chloride (Sigma-Aldrich, Steinheim, Germany) ( $\mathrm{pH} 7.4) .{ }^{36}$ The final incubations contained 1 $\mu \mathrm{M}$ substrate (final solvent concentration: $0.1 \%$ DMSO) in $100 \mathrm{mM}$ potassium phosphate buffer with $5 \mathrm{mM}$ magnesium chloride $(\mathrm{pH}$ 7.4), supplemented with $0.025 \mathrm{mg} / \mathrm{mL}$ of alamethicin (Sigma-Aldrich, Steinheim, Germany), $0.1,0.5$, or $1 \mathrm{mg} / \mathrm{mL}$ of HLS9 or RLS9, and 1 $\mathrm{mM}$ L-ascorbic acid (Sigma-Aldrich, Steinheim, Germany) to increase the stability of the substrate. After $5 \mathrm{~min}$ of preincubation, the reaction was started by adding a mix of three cofactors: NADPH (Roche Diagnostics, Mannheim, Germany), UDPGA (Sigma, Steinheim, Germany), and PAPS (Sigma, Steinheim, Germany) to allow both phase I and phase II reactions to take place. ${ }^{37}$ All (pre)incubations were carried out in Eppendorf tubes (Safe-Lock 1.5 $\mathrm{mL}$, Eppendorf) in a shaking incubator $(300 \mathrm{rpm})$ at $37{ }^{\circ} \mathrm{C}$ (Eppendorf Thermomixer C). The final reaction volume was $100 \mu \mathrm{L}$. Reactions were stopped by adding $100 \mu \mathrm{L}$ of ice cold methanol after $0,5,10,20,40$, or $60 \mathrm{~min}$ to the incubation vials. Samples were vortexed, put on ice, and stored at $-20{ }^{\circ} \mathrm{C}$. Two types of controls were included: incubations without $S 9$ fractions and incubations without the cofactor mix.

The incubations were analyzed using LC-MS to quantify the remaining parent compound in the incubations. To that end, samples were defrozen and centrifuged at $14000 \mathrm{rpm}$ at room temperature for $10 \mathrm{~min}$. Supernatant was transferred to glass insert vials suitable for LC-MS/MS injection (BGB Analytik Benelux B.V., Harderwijk, The Netherlands). More details on the LC-MS analysis can be found in the Supporting Information 2. A total of four replicates of the 

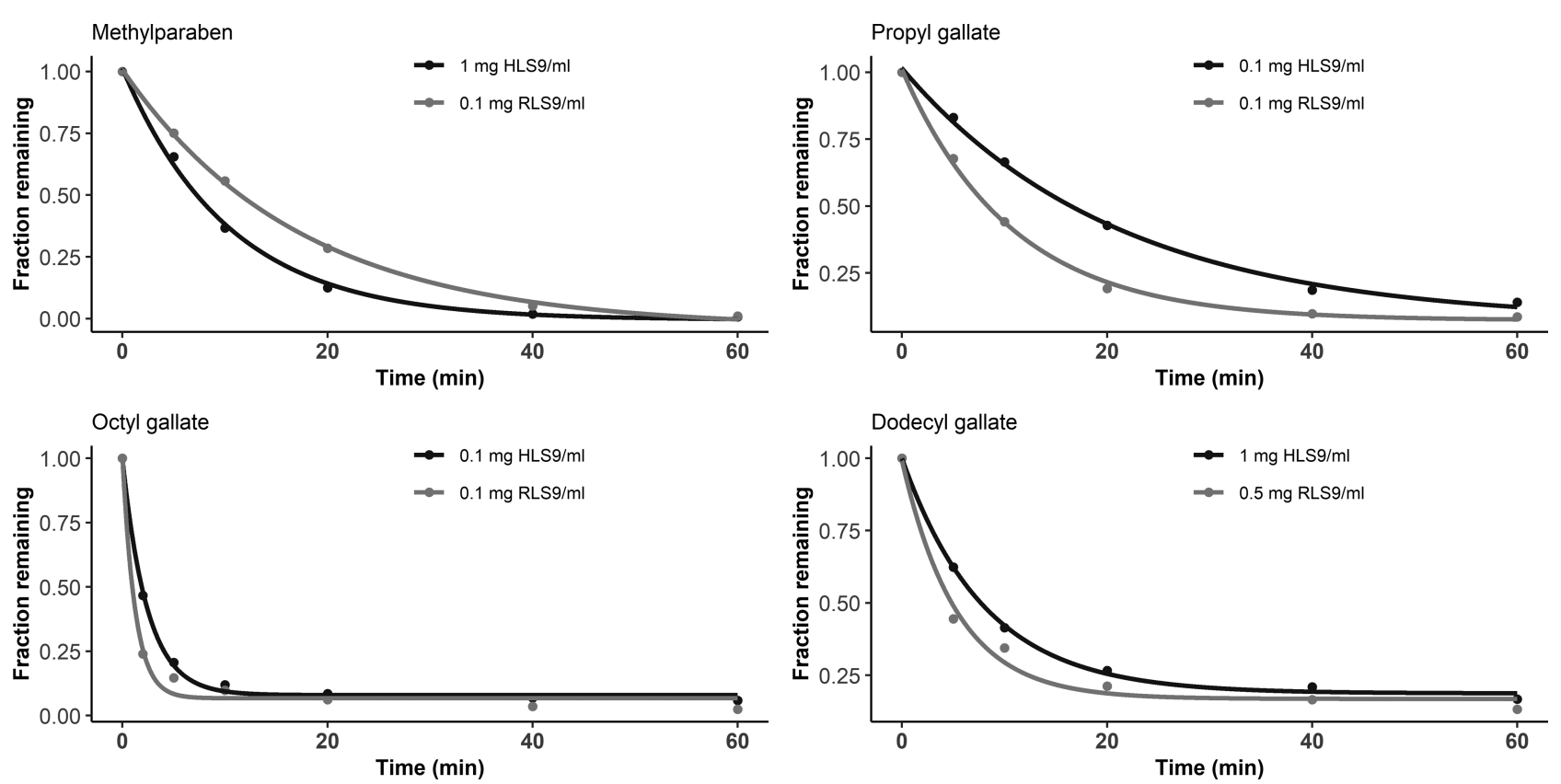

Figure 3. Substrate depletion of methylparaben, propyl gallate, octyl gallate, and dodecyl gallate in incubations with rat (gray lines and dots) or human (black lines and dots) liver S9. The dots correspond to the observed time-dependent fraction of the concentration measured at $t=0$ that remains in the incubation. For each experimental condition, the optimal $S 9$ concentration $(0.1,0.5$, or $1 \mathrm{mg} / \mathrm{mL})$ was determined in pilot studies (data not shown).

incubations were carried out, on two independent days (two replicates per day).

CLint values were determined by plotting the natural logarithm (ln) of the percentage of the remaining substrate (compared to $t=0$ ) against time. The slope of the linear part of these ln-transformed substrate-depletion curves represents the elimination rate constants $\left(k, \min ^{-1}\right) \cdot{ }^{37,38}$ After calculation of the half-life of each compound $\left(\mathrm{t} 1 / 2(\mathrm{~min})=\ln (2) / k\left(\mathrm{~min}^{-1}\right)\right)$ and volume of incubations $(\mathrm{V}(\mu \mathrm{L} /$ $\mathrm{mg})=1000 /[$ liver S9] $(\mathrm{mg} / \mathrm{mL})$, CLint was calculated by CLint $(\mu \mathrm{L} / \mathrm{min} / \mathrm{mg}$ of protein $)=\mathrm{V}(\mu \mathrm{L} / \mathrm{mg}) * \ln (2) / \mathrm{t} 1 / 2(\min ) .{ }^{39}$

2.3. In Silico Calculated Input Parameters. Partition coefficients, the fraction unbound in plasma, and the fraction unbound in the in vitro clearance incubation were estimated based on the $\log \mathrm{P}$ and $\mathrm{p} K_{\mathrm{a}}$ values of the chemicals by applying the calculators integrated in the web-based tool. The $\log \mathrm{P}$ and $\mathrm{p} K_{\mathrm{a}}$ values of the different compounds were derived from PubChem. For octyl gallate and dodecyl gallate, no $\mathrm{p} K_{\mathrm{a}}$ values were available from PubChem, and these values were taken from Tsao. ${ }^{40}$ For the calculations of the partition coefficients, either the method of Rodgers and Rowland ${ }^{28}$ or the method of Poulin and Theil that contains the correction by Berezhkovskiy ${ }^{29,41}$ can be selected in the web-based tool, as described in Section 2.1. In the present study, the calculator of the method of Poulin and Theil/Berezhkovskiy was used for calculating the partition coefficients of methylparaben and the gallates. Although the different methods provide different outcomes for the tissue:plasma partition coefficients, the choice of the calculator does not affect the conclusions of the study (data not shown). For the calculation of fraction unbound in plasma, one calculator is incorporated in the toolbox by using the method of Lobell and Sivarajah as described above. ${ }^{30}$ The fraction unbound in the in vitro S9 incubations is automatically calculated when entering information on the $S 9$ concentration applied in the in vitro incubation, based on a method of Hallifax and Houston ${ }^{34}$ for microsomal incubations, assuming the same unspecific binding in $S 9$ incubations compared to microsomal incubations. When clearance input is based on in vitro incubations with primary hepatocytes, the free fraction in the in vitro incubation is automatically calculated based on the method of Kilford et al. $^{33}$ for hepatocyte incubations based on the addition of the hepatocyte concentration applied in the in vitro incubations.
Regarding intestinal uptake, $k_{\mathrm{a}}$ values were estimated based on Caco-2 Papp values that were estimated based on TPSAs obtained from PubChem of the chemicals by applying eq $1 .^{42}$ The Papp values obtained were subsequently scaled to an uptake rate constant using eqs 2-4 for rat and human. These calculations were performed without the toolbox, and the resulting $k_{2}$ was included as an input in the web-based toolbox.

$$
\begin{aligned}
& \log \text { Papp }(\mathrm{cm} / \mathrm{s})=-4.36-0.01 * \text { TPSA } \\
& \log \text { Peff, human }\left(10^{-4} \mathrm{~cm} / \mathrm{s}\right) \\
& \quad=0.4926^{*} \log \text { Papp }\left(10^{-6} \mathrm{~cm} / \mathrm{s}\right)-0.1454
\end{aligned}
$$

Peff, rat $=$ Peff, human $/ 3.6$

$$
k_{\mathrm{a}}(/ \mathrm{h})=\text { Peff*2 }(\mathrm{cm} / \mathrm{s}) / \mathrm{R}(\mathrm{cm}) * 3600(\mathrm{~s} / \mathrm{h})
$$

in which eq 1 is derived from Hou et al. ${ }^{42}$ Eq 2 is an in-vitro-to-in-vivo scaler of the Caco-2 apparent permeability to an human effective permeability based on Sun et al., ${ }^{43}$ eq 3 scales the human effective permeability to rat effective permeability based on an equation that is derived from Fagerholm et al., ${ }^{44}$ and eq 4 describes how the effective permeabilities are converted to $k_{\mathrm{a}}$ as derived from $\mathrm{Yu}$ and Amidon. ${ }^{35}$ For the calculation of the rat and human uptake rates, intestinal radii $(R)$ of 0.18 and $1 \mathrm{~cm}$, respectively, were used. ${ }^{45}$

2.4. Determination of Oral Equivalent Doses Based on QIVIVE of ToxCast Data and Comparison of Oral Equivalent Doses with Exposure Data. To determine oral equivalent doses, it was assumed that (toxic) effects of methylparaben and the gallates are caused by the parent compounds and not by their metabolites. Therefore, in vitro toxicity data obtained with the parent compounds are required, and QIVIVE should be based on linking internal concentrations of the parent compound to in vitro effect concentrations. We determined oral equivalent doses based on available in vitro toxicity data from the ToxCast program. These in vitro biological activity data of methylparaben and the different gallates were derived from the ToxCast summary data, accessed through the EPA's CompTox Chemicals Dashboard. ${ }^{46}$ The AC50 values of all assays in which the compounds were active were downloaded from the Bioactivity (ToxCast:summary) tab. Differences 
Table 1. Chemical-Specific Characteristics and Input Parameters for the Web-Based Toolbox for PBK-Model Predictions

\begin{tabular}{|c|c|c|c|c|}
\hline parameter $^{a}$ & methylparaben & propyl gallate & octyl gallate & dodecyl gallate \\
\hline molecular weight $(\mathrm{g} / \mathrm{mol})$ & 152.15 & 212.2 & 282.33 & 338.4 \\
\hline CAS & $99-76-3$ & $121-79-9$ & $1034-01-1$ & $1166-52-5$ \\
\hline purity & $99.9 \%$ & $99.7 \%$ & $100 \%$ & $100 \%$ \\
\hline $\mathrm{p} K_{\mathrm{a}}^{b}$ & $8.5(\mathrm{~A})$ & $7.94(\mathrm{~A})$ & $7.94(\mathrm{~A})$ & $7.93(\mathrm{~A})$ \\
\hline $\log \mathrm{P}^{b}$ & 1.96 & 1.87 & 4.33 & 6.37 \\
\hline $\mathrm{CLint}_{\text {app }}(\mu \mathrm{L} / \mathrm{min} / \mathrm{mg}$ of $\mathrm{S} 9$ protein $)$ & $102(\mathrm{H}), 629(\mathrm{R})$ & $428(\mathrm{H}), 818(\mathrm{R})$ & $3119(\mathrm{H}), 3662(\mathrm{R})$ & $88(\mathrm{H}), 232(\mathrm{R})$ \\
\hline $\mathrm{mg}$ of $\mathrm{S} 9$ protein $/ \mathrm{mL}$ of incubation & $1(\mathrm{H}), 0.1(\mathrm{R})$ & $0.1(\mathrm{H}), 0.1(\mathrm{R})$ & $0.1(\mathrm{H}), 0.1(\mathrm{R})$ & $1(\mathrm{H}), 0.5(\mathrm{R})$ \\
\hline estimated fraction unbound in the incubation ${ }^{c}$ & $0.84(\mathrm{H}), 0.98(\mathrm{R})$ & $0.98(\mathrm{H}), 0.98(\mathrm{R})$ & $0.78(\mathrm{H}), 0.78(\mathrm{R})$ & $0.01(\mathrm{H}), 0.02(\mathrm{R})$ \\
\hline CLint,u $(\mu \mathrm{L} / \mathrm{min} / \mathrm{mg} \text { of } \mathrm{S} 9 \text { protein })^{d}$ & $121(\mathrm{H}), 642(\mathrm{R})$ & $437(\mathrm{H}), 835(\mathrm{R})$ & $3999(\mathrm{H}), 4695(\mathrm{R})$ & $8800(\mathrm{H}), 11600(\mathrm{R})$ \\
\hline TPSA $^{e}$ & 46.5 & 87 & 87 & 87 \\
\hline Papp $\left(10^{-6} \mathrm{~cm} / \mathrm{s}\right)^{f}$ & 15 & 6 & 6 & 6 \\
\hline Peff $\left(10^{-4} \mathrm{~cm} / \mathrm{s}\right)^{g}$ & $2.7(\mathrm{H}) 0.8(\mathrm{R})$ & $1.7(\mathrm{H}) 0.5(\mathrm{R})$ & $1.7(\mathrm{H}) 0.5(\mathrm{R})$ & $1.7(\mathrm{H}) 0.5(\mathrm{R})$ \\
\hline$k_{\mathrm{a}}(/ \mathrm{h})^{h}$ & $2.0(\mathrm{H}) 3.0(\mathrm{R})$ & $1.2(\mathrm{H}) 1.9(\mathrm{R})$ & $1.2(\mathrm{H}) 1.9(\mathrm{R})$ & $1.2(\mathrm{H}) 1.9(\mathrm{R})$ \\
\hline
\end{tabular}

${ }^{a} \mathrm{~A}$, acid; CLint ${ }_{\text {app}}$, apparent in vitro intrinsic clearance; $\log \mathrm{P}, \log$ partition coefficient between octanol and water; H, human; TPSA, topological polar surface area; $\mathrm{p} K_{\mathrm{a}}$, ionization coefficient; $\mathrm{R}$, rat. ${ }^{b}$ Obtained from PubChem (https://pubchem.ncbi.nlm.nih.gov/), except for $\mathrm{p} K_{\mathrm{a}}$ values for octyl gallate and dodecyl gallate, which were taken from Tsao. ${ }^{40}$ Fraction unbound in the in vitro incubation based on method by Hallifax and Houston $^{34}$ (calculated and integrated in PBK-model code by web-based toolbox). ${ }^{d}$ CLint ${ }_{\text {app }}$ corrected for the fraction unbound in the in vitro incubation (calculated and integrated in PBK-model code by web-based toolbox). ${ }^{e}$ Obtained from PubChem (https://pubchem.ncbi.nlm.nih.gov/) ${ }_{i}$ Calculated using TSPA as input in eq $1 .{ }^{42}{ }^{g}$ Calculated using estimated Papp value in eq 2 for humans (H) followed with eq 3 for rats (R). ${ }^{43,44}$ ${ }^{h}$ Calculated using estimated Peff in eq $4 .{ }^{35}$

occur in the number of positive hit calls (i.e., number of assays for which a $50 \%$ of maximum activity is reached, with its related effect concentration (AC50)). For example, methylparaben is active in 24 out of 886 assays in which it has been tested, whereas propyl gallate is active in 131 out of 888 assays, octyl gallate is active in 355 out of 982 assays, and dodecyl gallate is active in 210 out of 441 assays. Given that not all of the compounds have been tested in all assays, the differences in active hit calls cannot be interpreted as overall differences in bioactivity. ${ }^{13}$ Along with the biological activity data, the EPA's CompTox Chemicals Dashboard also provides so-called warning signs ("flags") to provide an indication of possible flaws in the data set, providing unreliable AC50 values and possible falsepositive or false-negative results. The data related to these flags were manually evaluated to conclude whether effects were considered to be real or false-positive.

For the determination of oral equivalent doses based on the ToxCast data, it was assumed that (1) toxicity/bioactivity in the in vivo situation is related to the maximal concentration ( $\mathrm{Cmax}$ ) reached in the plasma and (2) toxicity/bioactivity in the in vivo situation is related to the reported AC50 value in the ToxCast assay, expressed as the total (nominal) concentration in vitro. Obviously, this is an oversimplification of the reality, and as such, the approach in this example cannot be used to estimate effect dose levels that can be used to derive a point of departure for the risk assessment but should be seen as a low tier assessment that can be used to compare chemicals for prioritization for further assessment. For a higher tier assessment, one would need more information on the in vitro biokinetics, e.g., information on the free fraction and/or the cellular fraction responsible for the effect in the in vitro assay as well as full concentration-response data and not only a $50 \%$ effect concentration (AC50). Also, for a higher tier assessment based on such QIVIVE approaches, clear insight in the relevance of the in vitro method is required regarding the mechanisms it captures, preferably related to a key event of a relevant adverse outcome pathway (AOP). In the present study, we related the total concentration in vivo (Cmax) to the nominal effect concentration in vitro (AC50) for QIVIVE. If information on the free concentration in the in vitro assays would be available, one would preferably relate the free effect concentration in vitro to the unbound (free) Cmax in vivo for QIVIVE. As we have no information on the free concentrations for these chemicals in the ToxCast assays, we have chosen to also use the total concentration (instead of the unbound) concentration in vivo for QIVIVE, which can be considered to be a conservative approach, as the free fraction in vivo is in general lower than the free fraction in vitro, as protein and lipid concentrations in plasma are higher than in in vitro assays.

Oral equivalent doses obtained for humans were compared with information reported in the literature on estimated human exposure levels. These comparisons provide insight into whether humans are exposed to levels that are expected to reach internal concentrations that cause effects in the in vitro bioassays or for which chemical oral equivalent doses are closest to exposure levels, in order to prioritize chemicals for which further assessment is deemed necessary.

\section{RESULTS}

3.1. In Vitro and In Silico Input Data Used as Input for the PBK Models. Different input parameters were collected to predict plasma concentrations of methylparaben, propyl gallate, octyl gallate, and dodecyl gallate in rats and humans using the web-based toolbox. Figure 3 shows the substratedepletion curves of the compounds as measured in incubations with RLS9 and HLS9, applying the optimal S9 concentrations that were determined for each experimental situation (data not shown). The data are presented as fraction of remaining chemical at $t=0$. A starting concentration of $1 \mu \mathrm{M}$ was used, which is expected to be lower than the $\mathrm{Km}$ values of the reactions. It must be noted that the measured concentrations at $t=0$ were lower than the added $1 \mu \mathrm{M}$ for octyl gallate and dodecyl gallate (up to 2-fold lower), suggesting binding of the chemical to the plastic walls of the incubation tubes. As this was noticed to be stable in time in control incubations, the loss of chemical due to binding to plastic was considered to be instantaneous. As we assume that clearance is determined at concentrations lower than the $\mathrm{Km}$, the exact starting concentration available for metabolism $(0.5$ or $1 \mu \mathrm{M})$ is not critical as long as clearance values are obtained based on the data expressed as the fraction of the starting concentration that disappears in time.

Based on these substrate-depletion curves, the CLint values were calculated, which were used as input in the web-based toolbox. Both Figure 3 and the derived CLint values from these data (Table 1) reveal a swift conversion of the different compounds by both HLS9 and RLS9. Conversion was generally found to be faster by RLS9 than by HLS9, 
PBK-model result

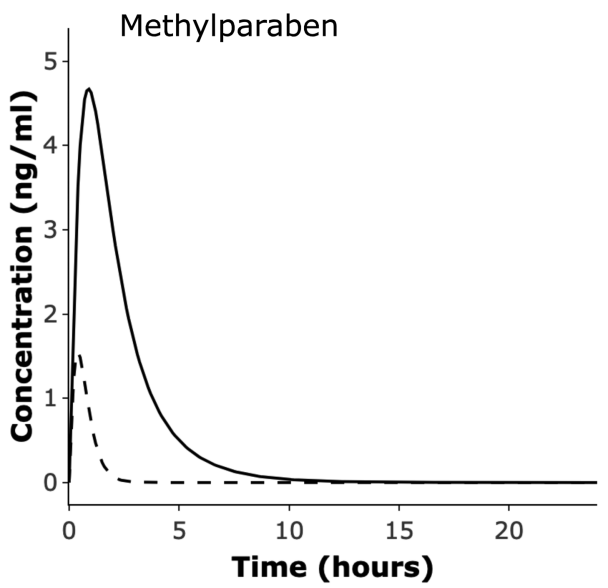

PBK-model result

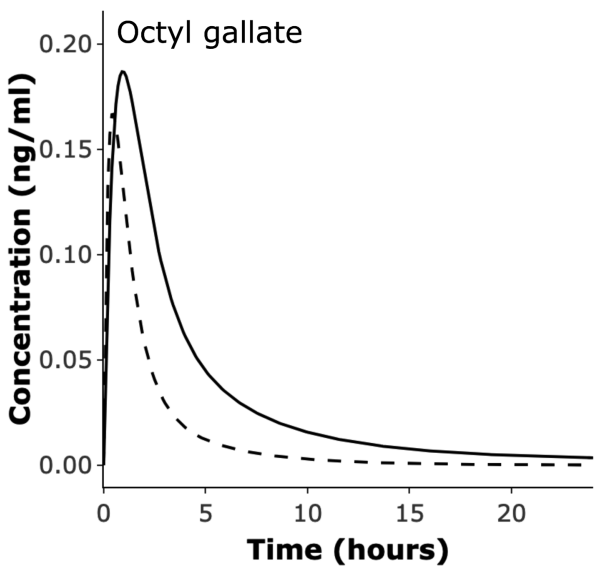

PBK-model result
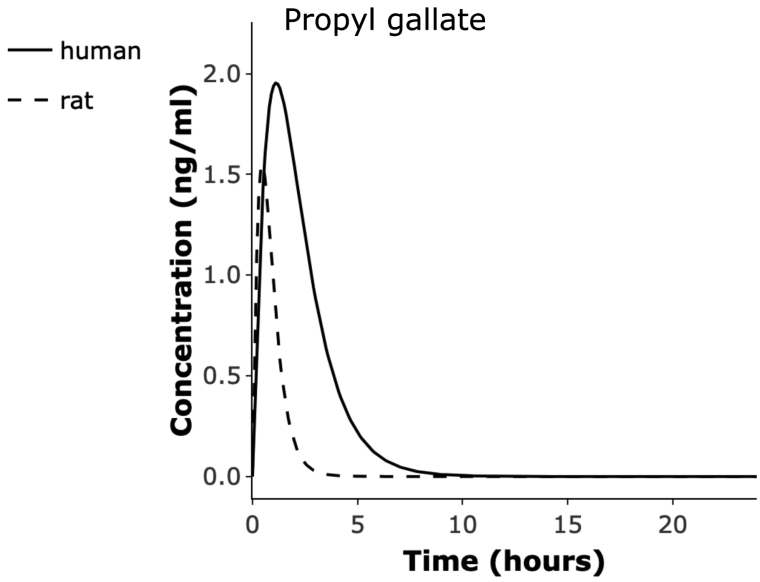

PBK-model result
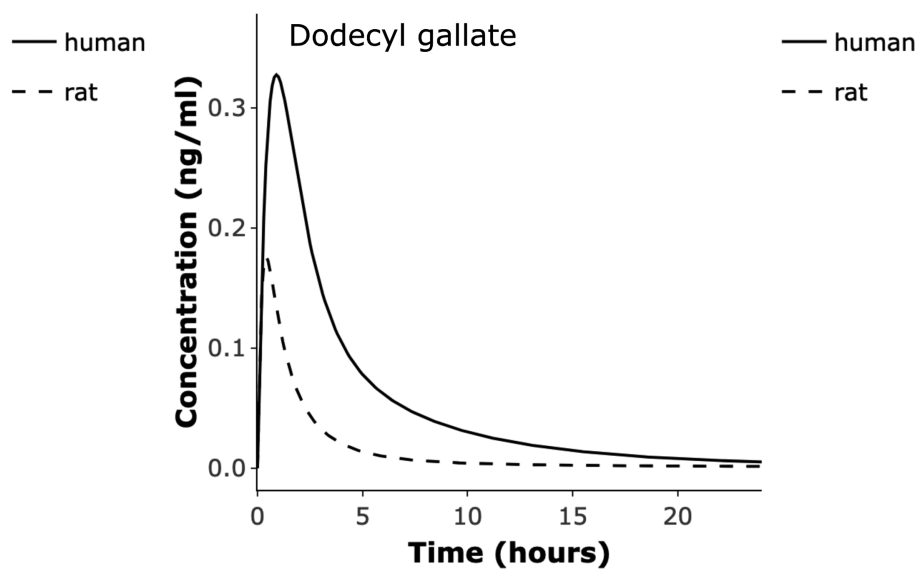

Figure 4. Screenshots of the simulated total (bound and unbound) plasma concentrations of methylparaben, propyl gallate, octyl gallate, and dodecyl gallate at $0.1 \mathrm{mg} / \mathrm{kg}$ bw in humans and rats using the web-based toolbox.

particularly in the case of methylparaben, which is 6-fold faster converted by RLS9 compared with HLS9. The highest CLint is observed for octyl gallate ( 3119 and $3662 \mu \mathrm{L} / \mathrm{min} / \mathrm{mg}$ of S9 protein by human and rat liver $\mathrm{S} 9$, respectively), whereas the lowest CLint is observed for dodecyl gallate (88 and $232 \mu \mathrm{L} /$ $\mathrm{min} / \mathrm{mg}$ of $S 9$ protein by human and rat liver $S 9$, respectively). These values are corrected for unspecific binding to the $S 9$ by the web-based toolbox (based on method of Hallifax and Houston $^{34}$ ), and the related CLint values for the unbound fraction (CLint, $\mathrm{u}$ ) are automatically applied in the PBK-model code for adequate predictions (tab "Metabolism" of PBK Workflow). The correction for unspecific binding to S9 particularly affects the results for dodecyl gallate (Table 1), showing that the CLint,u values for dodecyl gallate are even higher than for octyl gallate. Conversion of methylparaben (and to some extent of octyl and dodecyl gallate) occurs both in the presence and absence of the cofactor mix in the incubations with RLS9 and HLS9 (data not shown), suggesting that cofactor-independent metabolic routes are involved in the conversion. In case of methylparaben, hepatic esterases are known to be involved, which use $\mathrm{H}_{2} \mathrm{O}$ as cofactor. $^{13}$ The compounds are not converted in controls without S9 (data not shown).
In addition to the obtained CLint values, Table 1 provides an overview of the chemical-specific information and input parameters of the models, including the physicochemical characteristics of the compounds that are inserted in the webbased tool $\left(\log \mathrm{P}\right.$ and $\left.\mathrm{p} K_{\mathrm{a}}\right)$ and that are used to estimate Caco2 Papp values (TPSA) and corresponding $k_{\mathrm{a}}$ values. Notable differences between the compounds are in the $\log \mathrm{P}$ values, with particularly octyl gallate and dodecyl gallate being highly lipophilic.

3.2. Evaluation of the PBK-Model Predictions. Making use of the input parameters from Table 1 and the web-based tool, the plasma concentrations of each compound were simulated for rats and humans at different oral doses. Figure 4 shows the predictions of the total (bound and unbound) plasma concentrations of the four test chemicals as presented by the web-based toolbox at a single dose of $0.1 \mathrm{mg} / \mathrm{kg}$ body weight (bw). These PBK-model-based predictions provide important insights in possible differences in internal exposure in rats and humans. The largest differences in plasma concentrations between rats and humans at equal dose levels are observed for methylparaben. The observed 6-fold higher in vitro CLint of methylparaben in rats results in 3-fold lower plasma concentrations. Given that the scaled intrinsic clearance of methylparaben is higher than the blood flow to the liver in 

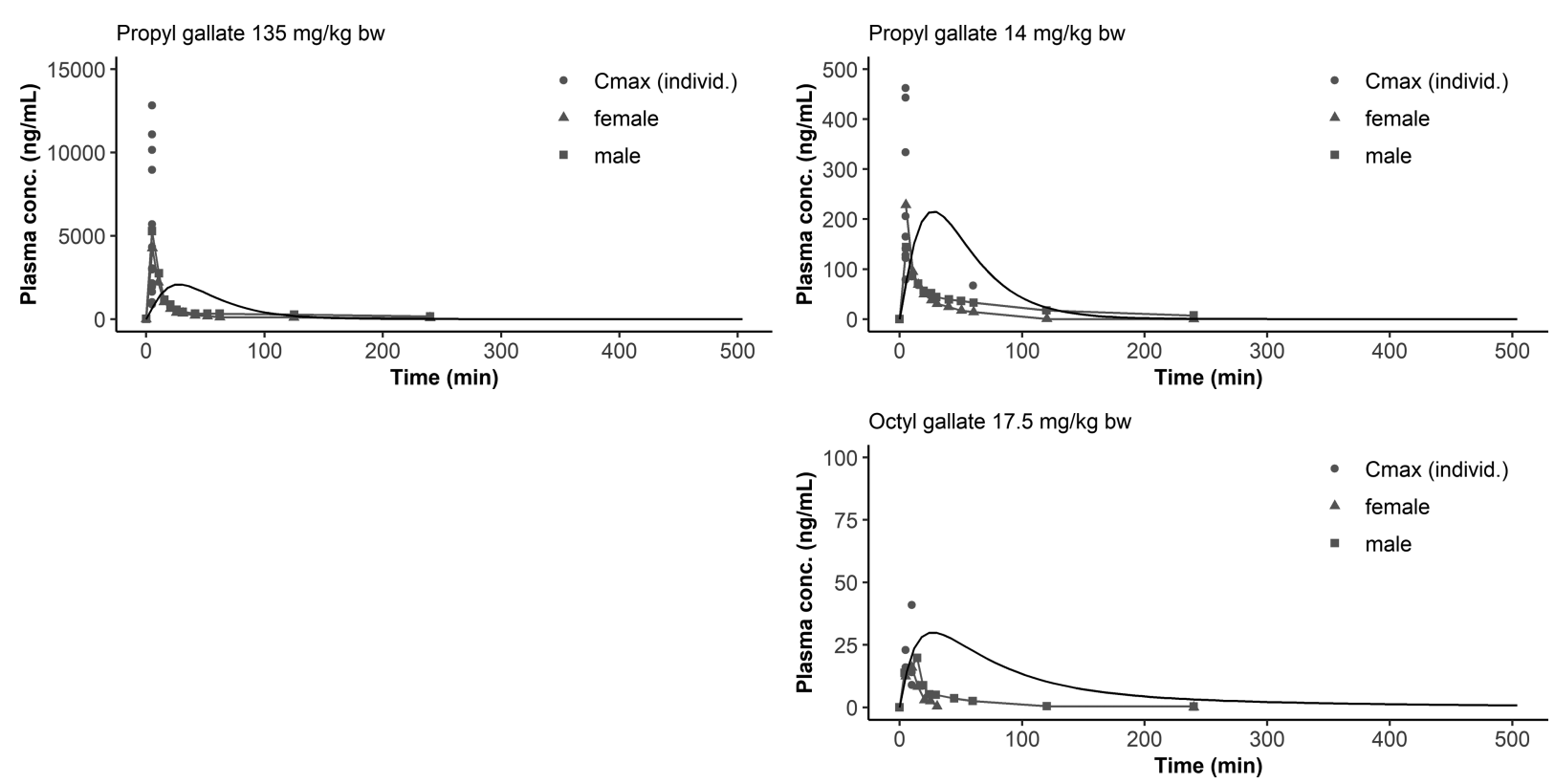

Figure 5. PBK-model-predicted (smooth line) and in vivo observed (symbols) plasma concentrations of propyl gallate and octyl gallate in rats at different oral doses. In vivo rat data were taken from Tullberg et $\mathrm{al}^{47}$ and represent reported average values for each sex of eight rats for $135 \mathrm{mg}$ propyl gallate $/ \mathrm{kg} \mathrm{bw}$, six rats for $14 \mathrm{mg}$ propyl gallate $/ \mathrm{kg}$ bw, and five rats for $17.5 \mathrm{mg}$ octyl gallate $/ \mathrm{kg}$ bw. More details on the in vivo and PBKmodel-predicted TK parameters (Cmax and AUC) are presented in Table 2.

Table 2. PBK-Model-Predicted and In Vivo-Reported Cmax and AUC in Plasma of Rats Exposed to Propyl Gallate, Octyl Gallate, Dodecyl Gallate, and Methylparaben and in Plasma of Humans Exposed to Propyl Gallate ${ }^{a, b}$

\begin{tabular}{|c|c|c|c|c|c|}
\hline & \multirow[b]{2}{*}{ exposure } & \multicolumn{2}{|c|}{ PBK-model prediction } & \multicolumn{2}{|c|}{ reported in vivo } \\
\hline & & $\mathrm{Cmax}(\mathrm{ng} / \mathrm{mL})$ & $\operatorname{AUC}(\mathrm{ng} * \mathrm{hr} / \mathrm{mL})$ & $\mathrm{Cmax}(\mathrm{ng} / \mathrm{mL})$ & AUC (ng*hr/mL) \\
\hline \multirow[t]{5}{*}{ rats } & $135 \mathrm{mg}$ of propyl gallate $/ \mathrm{kg} \mathrm{bw}$ & 2089 & 2333 & $\begin{array}{l}\text { ㅇ } 4246(884-12825) n=8 \\
\text { o } 5260(1635-11085) n=8 \\
\text { 우 and } \delta 4753 n=16\end{array}$ & $\begin{array}{l}\text { ㅇ } 1164(321-3451) n=8 \\
\text { o } 2085(660-4119) n=8 \\
\text { 우 and } ð 1624 n=16\end{array}$ \\
\hline & $14 \mathrm{mg}$ of propyl gallate $/ \mathrm{kg}$ bw & 217 & 242 & $\begin{array}{l}\text { ㅇ } 238(68-462) n=6 \\
\text { o } 145(67-334) n=6 \\
\text { 우 and } \delta 192 n=12\end{array}$ & $\begin{array}{l}\text { ㅇ } 66(39-100) n=6 \\
\text { o } 123(62-217) n=6 \\
\text { 우 and } \delta 95 n=12\end{array}$ \\
\hline & $17.5 \mathrm{mg}$ of octyl gallate $/ \mathrm{kg} \mathrm{bw}$ & 29 & 60 & $\begin{array}{l}\text { ㅇ } 20\left(\text { range NR) }{ }^{\mathrm{a}}\right. \\
\text { o } 19(9-41) n=6\end{array}$ & $\begin{array}{l}\text { ㅇ NR } \\
\text { o } 3.7(1.4-7.8) n=6\end{array}$ \\
\hline & $10 \mathrm{mg}$ of dodecyl gallate $/ \mathrm{kg} \mathrm{bw}$ & 18 & 38 & ND & $\mathrm{ND}$ \\
\hline & $100 \mathrm{mg}$ of methylparaben $/ \mathrm{kg} \mathrm{bw}$ & 1544 & 1436 & ND & $\mathrm{ND}$ \\
\hline \multirow[t]{2}{*}{ humans } & $14 \mathrm{mg}$ of propyl gallate $/ \mathrm{kg} \mathrm{bw}$ & 274 & 770 & $\begin{array}{l}\text { ㅇ } 150(10-240) n=5 \\
o 155(84-208) n=5 \\
\text { ㅇ and } \delta 152 n=10\end{array}$ & $\begin{array}{l}\text { ㅇ } 290(25-425) n=5 \\
\text { o } 402(290-587) n=5 \\
\text { ㅇ and } ð 346 n=10\end{array}$ \\
\hline & $1.4 \mathrm{mg}$ of propyl gallate $/ \mathrm{kg}$ bw & 27 & 77 & $\begin{array}{l}\text { o } 12(11-14) n=4 \\
\text { o } 14(4-33) n=7 \\
\text { o and } \lesssim 13 n=11\end{array}$ & $\begin{array}{l}\text { o } 19(13-21) n=4 \\
\text { o } 21(9.4-42) n=7 \\
\text { o and } \delta 21 n=11\end{array}$ \\
\hline
\end{tabular}

${ }^{a} \mathrm{ND}$, not detected; NR, not reported. ${ }^{b}$ For in vivo data, mean values and ranges (min-max) are presented along with the number of individuals $(n)$.

rats and humans, the blood flow will be the rate limiting step in the conversion of methylparaben and the maximum difference in CLint cannot be reached in vivo. Differences in maximal plasma concentrations ( $\mathrm{Cmax}$ ) at equal dose levels between humans and rats are 1.3-fold for propyl gallate, 1.1-fold for octyl gallate, and 1.8-fold for dodecyl gallate. At a dose of 0.1 $\mathrm{mg} / \mathrm{kg}$ bw, methylparaben and propyl gallate reach higher plasma concentrations (expressed as $\mathrm{ng} / \mathrm{mL}$ ) than octyl gallate and dodecyl gallate (Figure 4).

To evaluate the model performance, the model predictions were compared with available in vivo kinetic data. Since QIVIVE in this study is based on the peak plasma concentrations $(\mathrm{Cmax})$ of the compounds, gaining adequate predictions of the Cmax values is deemed most important. Figure 5 shows the predicted and observed plasma concentrations of propyl gallate and octyl gallate at different oral doses in rats. Table 2 provides an overview of the Cmax and AUC values predicted by the PBK model for these exposures in rats and presents the reported Cmax and AUC values from the in vivo studies from Tullberg et al., ${ }^{47}$ also including PBK-model predictions of dodecyl gallate and methylparaben. Figure 5 and Table 2 reveal the relatively large variation in observed plasma concentrations of propyl gallate and octyl gallate between individual animals (as reported by Tullberg et al.). ${ }^{47}$ For example, in rats exposed to $135 \mathrm{mg}$ of propyl gallate $/ \mathrm{kg}$ bw, the observed Cmax values 

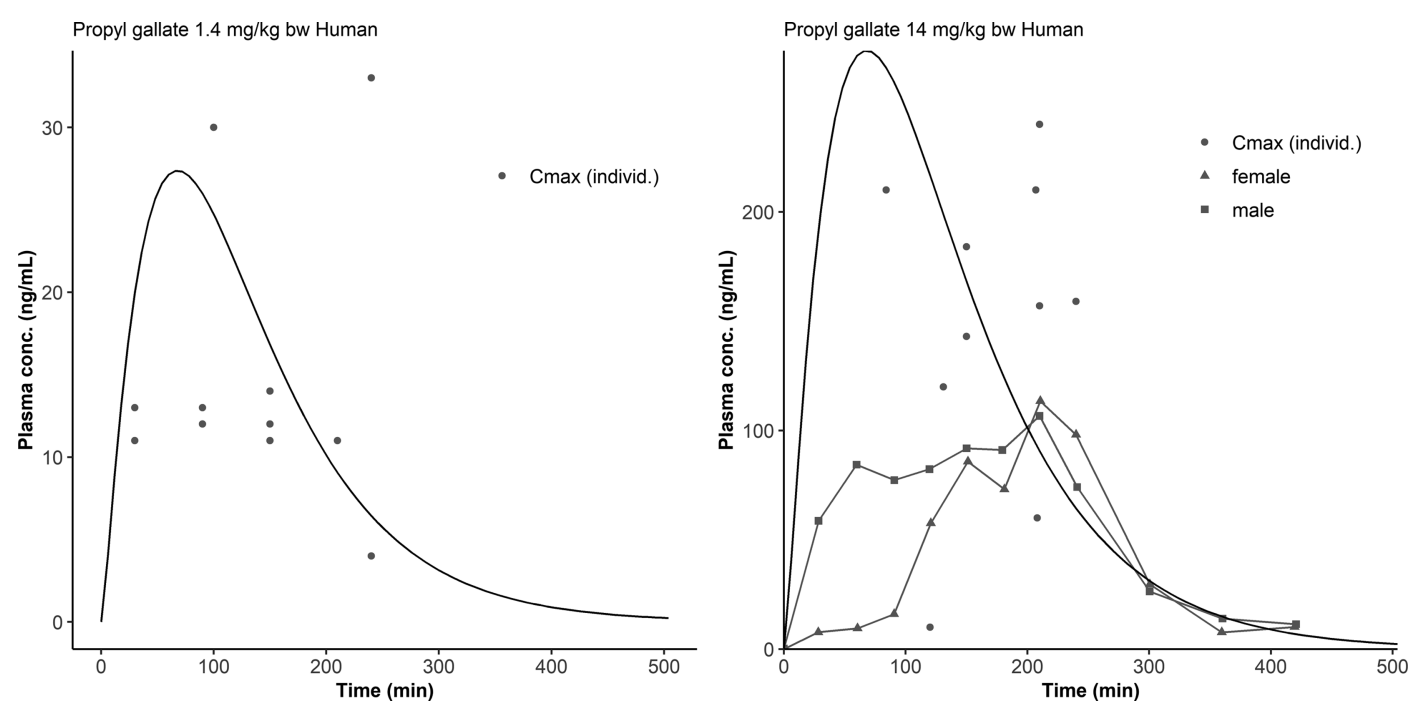

Figure 6. PBK-model-predicted (smooth line) and in vivo observed (symbols) plasma concentrations of propyl gallate in humans at different oral doses. In vivo human data were taken from Tullberg et al. ${ }^{47}$ and represent reported average values for each gender of five persons for $14 \mathrm{mg}$ of propyl gallate $/ \mathrm{kg}$ bw and four females and seven males for $1.4 \mathrm{mg}$ of propyl gallate $/ \mathrm{kg}$ bw. More details on the in vivo and PBK-model-predicted TK parameters (Cmax and AUC) are presented in Table 2.

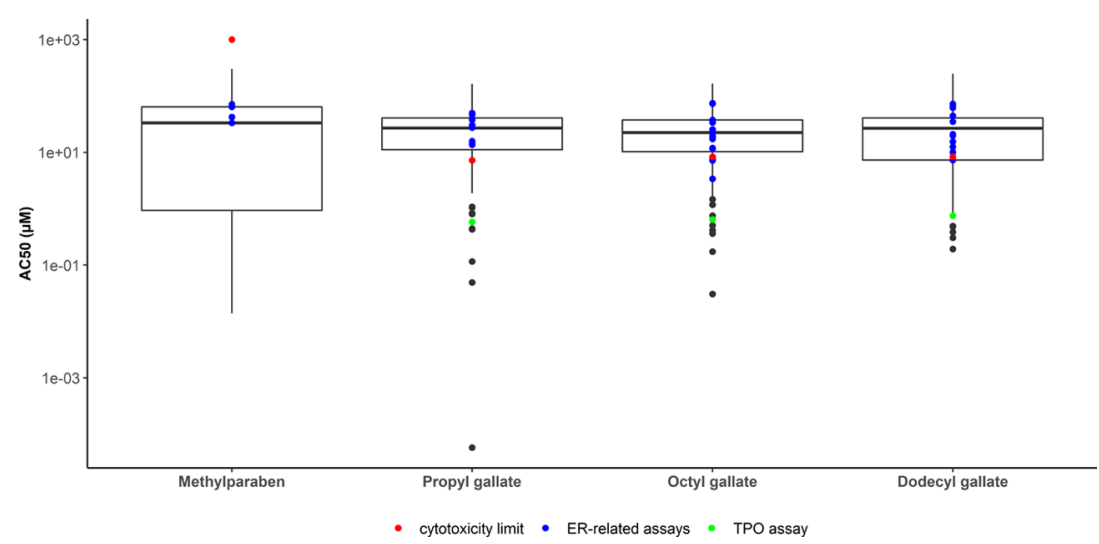

Figure 7. Box $(25-75$ th percentile $)$ and whisker $(1.5 \times \mathrm{IQR})$ plots, depicting the range of AC50 values of the chemicals within the ToxCast data set. The AC50 values for ER-related assays (blue dots), the ToxCast-reported cytotoxicity limits (red dots), and the NCCT_TPO_AUR_dn assay (green dots) are highlighted within the graph. Methylparaben has not been tested in the NCCT_TPO_AUR_dn assay. Black dots represent assays with AC50 values lower than the $1.5 \times \mathrm{IQR}$ whisker.

range between 884 and $12825 \mathrm{ng} / \mathrm{mL}$. The predicted Cmax of $2089 \mathrm{ng} / \mathrm{mL}$ at a dose of $135 \mathrm{mg} / \mathrm{kg}$ bw falls within this range. The same is true for the predicted Cmax of $217 \mathrm{ng} / \mathrm{mL}$ at a dose of $14 \mathrm{mg}$ of propyl gallate/kg bw, which falls within the observed range of 67 to $462 \mathrm{ng} / \mathrm{mL}$ at this oral dose. In the case of octyl gallate, the predicted Cmax of $29 \mathrm{ng} / \mathrm{mL}$ at a dose of $17.5 \mathrm{mg} / \mathrm{kg}$ bw is also within the observed Cmax values, ranging from 9 to $41 \mathrm{ng} / \mathrm{mL}$. Though the model predicts a fast disappearance of propyl gallate and octyl gallate from plasma, the observed disappearance appears to be even faster. Therefore, plasma concentrations of propyl gallate and octyl gallate at later time points (up to $120 \mathrm{~min}$ ) are somewhat overestimated in rats by the PBK model. A direct comparison between predicted and observed Cmax values is not straightforward for methylparaben and dodecyl gallate. Available in vivo data for rats exposed to an oral dose of 10 $\mathrm{mg}$ of dodecyl gallate $/ \mathrm{kg}$ bw revealed no detectable plasma concentrations in samples that were taken 10 min after dosing, which was the only time point at which blood was collected in the study with this chemical by Tullberg et al. ${ }^{47}$ The PBK- model-predicted plasma concentration at $10 \mathrm{mg}$ of dodecyl gallate $/ \mathrm{kg}$ bw amounts to $18 \mathrm{ng} / \mathrm{mL}$ (10 min after exposure), which confirms that plasma concentrations of dodecyl gallate are indeed low at this oral dose and close to the reported detection limit of 5-10 ng/mL. Methylparaben kinetics in rats were studied by Aubert et al., ${ }^{48}$ in which rats were exposed to $100 \mathrm{mg}$ of $\left[{ }^{14} \mathrm{C}\right]$-methylparaben $/ \mathrm{kg}$ bw. No parent compound was detected in the plasma at different time points up to $24 \mathrm{~h}$ after exposure. ${ }^{49}$ The PBK model predicts a Cmax of $1544 \mathrm{ng} /$ $\mathrm{mL}$ at a dose of $100 \mathrm{mg} / \mathrm{kg}$, but since no detection limit of the parent compound is provided by Aubert et al., ${ }^{48}$ it is even more difficult to evaluate whether the PBK-model predictions are adequate for methylparaben.

Regarding humans, only kinetic data are available on propyl gallate and not for the other chemicals. The PBK-modelpredicted plasma concentrations of propyl gallate in humans were evaluated against these available human experimental data (Figure 6; Table 2). At a dose of $1.4 \mathrm{mg} / \mathrm{kg}$, the predicted Cmax of $27 \mathrm{ng} / \mathrm{mL}$ falls within the observed range of Cmax values ( 4 to $33 \mathrm{ng} / \mathrm{mL}$ ). At a dose of $14 \mathrm{mg} / \mathrm{kg}$, the predicted 


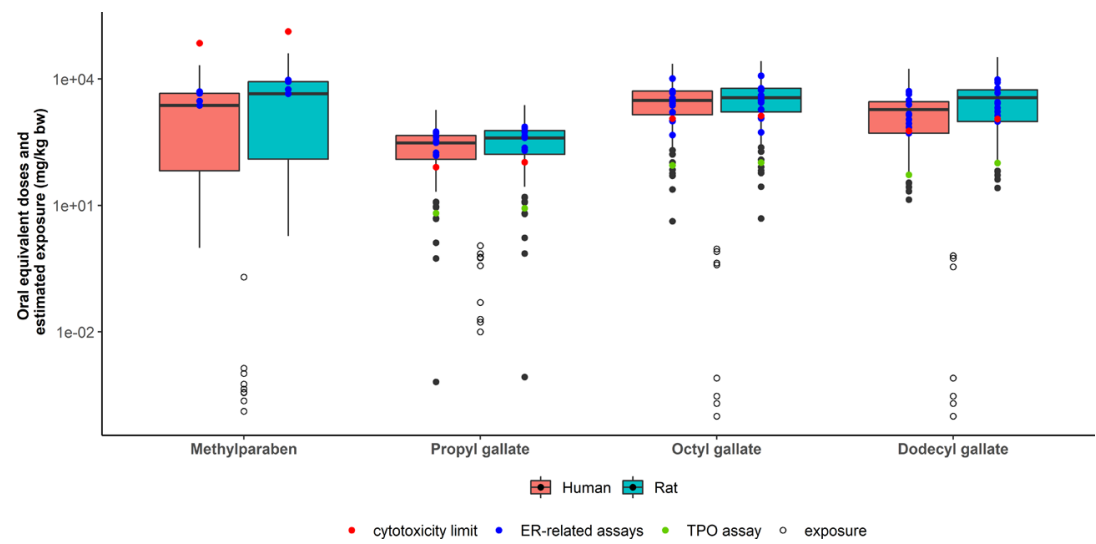

Figure 8. Box (25-75th percentile) and whisker $(1.5 \times \mathrm{IQR})$ plots depicting the range of oral equivalent doses of the AC50 values (Figure 7$)$ of the chemicals within the ToxCast data set and comparison of these oral equivalent doses to oral daily intake estimates (open dots). ${ }^{8,16,18,19}$ The oral equivalent doses related to ER-related assays (blue dots), the ToxCast-reported cytotoxicity limit (red dots), and the TPO inhibition assay (green dots) are highlighted within the graph.

Cmax of $274 \mathrm{ng} / \mathrm{mL}$ somewhat overestimates the observed Cmax values, which ranged from 10 to $240 \mathrm{ng} / \mathrm{mL}$.

Overall, the results of the model evaluation for the different compounds suggest that the model simulations are considered acceptable as first estimates of the Cmax in rats and humans, which is deemed most relevant for QIVIVE in the present study that is based on Cmax concentrations in plasma. Furthermore, although not required for QIVIVE in the present study, it is of interest to note that despite the slide overestimation of the AUC, the PBK-model-based predictions of the AUC remain within a 1.4 to 8 -fold range from the reported AUC values in rats and a 2- to 4-fold range from the reported AUC values in humans and can be considered acceptable.

Further optimization of the PBK model would lead to a better fit to the in vivo data, particularly with respect to time of the internal peak exposure (Tmax). It is of interest to note that the Tmax in rats is very low according to the in vivo data, which may indicate immediate uptake for these chemicals in the stomach. Uptake in the stomach is not described in the current PBK model, resulting in a higher PBK-model-predicted Tmax. The PBK model predicts a higher Tmax in humans than in rats, but the PBK-model-predicted Tmax for propyl gallate in humans is lower than indicated by the in vivo data. This may indicate a limited release of propyl gallate from the matrix in the human study, a process that is not described in the PBK model. It may also be related to a slower GI transit than the default transit time that was included in the model.

3.3. In Vitro ToxCast Data. In vitro biological activity data of methylparaben and the different gallates were derived from the ToxCast summary data, accessed through the EPA's chemistry dashboard (see Materials and Methods). In Figure 7, the ranges of in vitro (nominal) AC50 values for all assays in which the compounds are active are displayed as box and whisker plots. The individual AC50 values for estrogen receptor (ER)-related targets are highlighted in Figure 7, as the ER is frequently reported as a biological target of parabens. 8

The majority of the AC50 values (25-75th percentile boxes in Figure 7) fall within the range of $6.5-64 \mu \mathrm{M}$ for methylparaben, $11-41 \mu \mathrm{M}$ for propyl gallate, $10-37 \mu \mathrm{M}$ for octyl gallate, and $8.8-41 \mu \mathrm{M}$ for dodecyl gallate. The AC50 values for ER-related assays also predominantly fall within this range, with propyl gallate, octyl gallate, and dodecyl gallate having similar potencies, ranging between 22 and $47 \mu \mathrm{M}$. Methylparaben is slightly less active in ER-related assays, with AC50 values ranging between 42 and $71 \mu \mathrm{M}$. For propyl gallate, octyl gallate, and dodecyl gallate, a few AC50 values fall below the lower whisker, corresponding to the $1.5 \times$ interquartile range $(1.5 \times \mathrm{IQR})$ between the first and third quartile as the cut off for outliers. ${ }^{50}$ These in vitro end points with relatively low effect concentrations might be of particular interest, as the targets represented by these assays could potentially be affected at low exposure levels. These effect concentrations are also below the ToxCast-reported cytotoxicity limit. In-depth analysis of the concentration-response data of the assays with these relatively low effect concentrations revealed that the majority of these effects can be considered as irrelevant (i.e., no clear concentration-response curves or the presence of so-called flags that warn for potential problems with the underlying data, as reported in the EPA's chemistry dashboard). These assay results were not further considered for the QIVIVE as described below. Regarding these relative sensitive assays for these chemicals, the assay results were only considered relevant for the NCCT TPO AUR dn assay (green dots in Figure 7), as clear concentration- $\bar{d}$ ependent inhibition of TPO activity was observed, and no warning flags were connected to these data. Methylparaben has not been tested in the NCCT_TPO_AUR_dn assay.

3.4. Comparison of the QIVIVE-Based Oral Equivalent Doses in Rats and Humans with Human Oral Exposure

Data. Based on the PBK-model predictions, oral equivalent doses were calculated, being the doses that lead to Cmax values in plasma that are equal to the in vitro effect concentrations (AC50 values as also presented in Figure 7). The estimated oral equivalent doses related to the ToxCast AC50 values were compared with oral exposure estimates as derived from the different EFSA opinions of the compounds ${ }^{16,18,19}$ and from Brand et al. ${ }^{8}$ (Figure 8). The range of exposure values in Figure 8 correspond to the lowest and highest estimated exposure levels for toddlers, children, adolescents, and adults.

Oral equivalent doses for propyl gallate are lower than those of the other gallates (dodecyl gallate and octyl gallate) but within the same range as those for methylparaben (Figure 8). For all compounds, humans are predicted to be more sensitive 
than rats, which is due to the higher predicted plasma concentrations at equal dose levels (Figure 4). The majority of the activity data $(25-75$ th percentile boxes in Figure 8 ) is estimated to be elicited at $21-206 \mathrm{mg} / \mathrm{kg}$ bw for methylparaben, $119-443 \mathrm{mg} / \mathrm{kg}$ bw for propyl gallate, $1520-5624 \mathrm{mg} / \mathrm{kg}$ bw for octyl gallate, and $910-4240 \mathrm{~kg}$ bw for dodecyl gallate in humans. Reported human exposure ranges from 0.00013 to $0.2 \mathrm{mg} / \mathrm{kg}$ bw/day for methylparaben, from 0.01 to $1.11 \mathrm{mg} / \mathrm{kg} \mathrm{bw} /$ day for propyl gallate, from 0.0001 to $0.93 \mathrm{mg} / \mathrm{kg} \mathrm{bw} /$ day for octyl gallate, and from 0.0001 to $0.64 \mathrm{mg} / \mathrm{kg} \mathrm{bw} /$ day for dodecyl gallate. ${ }^{8,16,18,19}$ Comparison of the oral exposure estimates to the oral equivalent doses shows that for all compounds, human exposure generally remains below the oral equivalent doses related to most ToxCast assays (i.e., exposure remains below the range of the box and whisker plot).

Regarding the most sensitive ToxCast assay for these chemicals, the NCCT_TPO_AUR_dn assay, the results show that particularly for propyl gallate, the margin between human exposure and the oral equivalent dose related to TPO inhibition as measured with the NCCT_TPO_AUR_dn assay is relatively small. The AC50 value of propyl gallate in the NCCT TPO AUR dn assay amounts to $0.58 \mu \mathrm{M}$, which is estimated to be reached at $6.3 \mathrm{mg}$ of propyl gallate $/ \mathrm{kg}$ bw in humans and at $7.9 \mathrm{mg} / \mathrm{kg}$ bw in rats. The highest exposure estimate reported by EFSA for propyl gallate corresponds to $1.1 \mathrm{mg} / \mathrm{kg} \mathrm{bw}$ for adults. ${ }^{16}$ For toddlers and small children, the highest exposure has been estimated to amount to $0.57-0.59$ $\mathrm{mg} / \mathrm{kg} \mathrm{bw}{ }^{16}$ These exposure levels are 6- to 11 -fold lower than the oral equivalent dose in humans related to TPO inhibition as measured with the NCCT_TPO_AUR_dn assay. Octyl gallate and dodecyl gallate are also active in the TPO assay, but the oral equivalent doses related to TPO inhibition are higher for these compounds than for propyl gallate, amounting to 99 $\mathrm{mg} / \mathrm{kg}$ bw for octyl gallate and $69 \mathrm{mg} / \mathrm{kg}$ bw for dodecyl gallate. The margin between human exposure and these oral equivalent doses is at least 106-fold for octyl gallate and 122fold for dodecyl gallate. Given that methylparaben has not been tested in the NCCT_TPO_AUR_dn assay, no oral equivalent dose related to TPO inhibition could be derived for this chemical.

\section{DISCUSSION}

The objective of the present study was to develop a userfriendly web-based toolbox containing generic PBK models for rats and humans and underlying calculation tools for distribution parameters, which can be applied for performing QIVIVE. As a case study, the tool was used to determine oral equivalent doses related to in vitro ToxCast bioactivity data for the food additives methylparaben, propyl gallate, octyl gallate, and dodecyl gallate. These oral equivalent doses were subsequently compared with human exposure estimates to assess whether in vitro bioactivity can be expected at human relevant exposure. These results revealed that daily intake levels of especially propyl gallate can lead to internal plasma concentrations that are close to in vitro biological effect concentrations, particularly with respect to the inhibition of human thyroid peroxidase (TPO).

Although various freely available tools are available for PBKmodel development, including for example PK-SIM (http:// www.open-systems-pharmacology.org), PLETHEM, ${ }^{51} \mathrm{Httk}^{52}$ and TK Plate, ${ }^{53}$ the key goal of the present study was to develop a toolbox that can be used without PBK-modeling software and without basic understanding of and experience with $\mathrm{PBK}$ modeling or $\mathrm{R}$ programming. In order to make the toolbox as simple as possible, the PBK models for rats and humans that are included were built based on minimal input, being the $\log \mathrm{P}$ and $\mathrm{p} K_{\mathrm{a}}$ of the chemical (required for the calculations on tissue binding and for the calculation of the free fraction in plasma and in vitro hepatocyte/liver tissue incubations), a value for hepatic clearance, and passive intestinal uptake (which can be estimated based on the TPSA of the chemical) using a multiple compartment uptake model. $^{25,27}$ In contrast to other PBK-modeling tools, the in silico calculations for the partition coefficients, ${ }^{28,29,41}$ fraction unbound plasma, ${ }^{30}$ and free fraction in in vitro incubations with microsomes, ${ }^{34}$ S9 (assumed in this study to be similar as for microsomal incubations), or hepatocytes ${ }^{33}$ can be carried out independently of the PBK-model simulations, providing an easy and user-friendly tool for quick in silico calculations of chemical-specific PBK-model parameters, that can also be used as input for the user's own PBK models. We aim to extend the toolbox in the future with more of such calculation methods, including for example tools to calculate the free fraction in in vitro toxicity assays. 54,55

The minimal PBK models that are included in the toolbox will not be applicable to all chemicals. Generally, adequate estimations of in vivo kinetic parameters (particularly Cmax) can be made for chemicals that are rapidly absorbed and when liver metabolism is the main clearance route; ${ }^{37,38,56,57}$ however, this is not the case for chemicals that will largely depend on, for example, extrahepatic metabolism and/or active-transporter-mediated kinetics. Without data to evaluate model performance, one should therefore be cautious when applying the model for different chemicals. Apart from these limitations in the applicability domain, there are also restrictions with respect to scenarios that can be simulated with the toolbox. For example, only predictions of the parent chemical can be made, and the toolbox cannot be applied for QIVIVE of chemicals for which the toxicity is caused by the metabolites. In addition, predictions at relatively high dose levels may not be adequate, as no saturation of biotransformation enzymes is included. Finally, the models only provide predictions for an average adult human $(70 \mathrm{~kg})$ or rat $(0.25 \mathrm{~kg})$ and not for specific life stages or subpopulations (based on specific isoforms for biotransformation enzymes). Many of these above aspects like the saturating metabolism, extrahepatic, and isoenzyme-specific metabolism are relevant to be included in future updates of the toolbox. However, at present, the webtool should mainly be used to make first estimations that are useful in low tier assessments. When more advanced predictions are needed, the results can be used as a step-up to further PBK-model development in, for example, the freely available tools mentioned above, or GastroPlus (https://www.simulationsplus.com/software/gastroplus) or Simcyp (https://www. certara.com/software/simcyp-pbpk). In addition, workflows are available for probabilistic QIVIVE, allowing to account for uncertainties in the predicted oral equivalent doses of in vitro effect concentrations. ${ }^{58}$ The $\mathrm{R}$ code for the current PBK model underlying the toolbox is provided in Supporting Information 1 , allowing also further extension of the model in $\mathrm{R}$.

For the application of the toolbox, it should also be kept in mind that each of the included calculators for the partition coefficients, ${ }^{28,29,41}$ fraction unbound plasma, ${ }^{30}$ and the free fraction in in vitro incubations with microsomes ${ }^{34}$ or hepatocytes ${ }^{33}$ may have restrictions with respect to their 
applicability domain. The global performance of these different calculators has not been evaluated so far, and no recommendations can as yet be provided on the use of these calculators. Actions toward standardization of methods and development of guidance documents with related technical guidances for the different approaches are needed to obtain robust and reliable chemical-specific input parameters. ${ }^{59-61}$ This is a prerequisite for enhancing the ultimate use and acceptance of these different calculators and PBK models in toxicological research and for regulatory purposes.

The model evaluation against available in vivo data revealed that the Cmax values of propyl gallate and octyl gallate in rats and of propyl gallate in humans were adequately predicted by the PBK model. However, plasma concentrations of these compounds at time points following the Cmax (up to 120 min) were overestimated. In addition, the models did not adequately capture the time of the peak concentration (Tmax). A reduced fraction absorbed as a result of insolubility, a lack of inclusion of intestinal metabolism, different intestinal uptake kinetics related to active uptake and/or secretion processes, or uncertainties in the renal clearance (currently described as the glomerular filtration rate times the free concentration in plasma) might be underlying causes of the observed differences. Given the relative short half-life of methylparaben and the different gallates in plasma, the Cmax of these compounds was considered the most relevant internal exposure marker for the QIVIVE of the ToxCast data. Hence, any deviations between predicted and observed Tmax and the AUC were not considered critical to be further resolved in this study.

Based on the extrapolation of the ToxCast in vitro effect data, the majority of biological activities of methylparaben and the different gallates were estimated to occur at oral doses that are more than 2 orders of magnitude higher than the estimated daily intake levels of the compounds. Biological activity toward the TPO NCCT_TPO_AUR_dn assay, assessing inhibition of TPO, was predicted to occur at doses that are closer to human exposure levels, particularly in the case of propyl gallate. The oral equivalent dose related to TPO inhibition by propyl gallate was estimated to amount to $6.3 \mathrm{mg}$ of propyl gallate $/ \mathrm{kg}$ bw in humans and to $7.9 \mathrm{mg} / \mathrm{kg}$ bw in rats, whereas human exposure to propyl gallate has been estimated to amount to $0.1-1.11 \mathrm{mg} / \mathrm{kg} \mathrm{bw} /$ day. ${ }^{16}$ The predicted oral equivalent dose for propyl-gallate-induced TPO inhibition in rats of $7.9 \mathrm{mg} / \mathrm{kg}$ $\mathrm{bw} /$ day is lower than available NOAELs for propyl gallate from animal experimental data amounting to $150 \mathrm{mg} / \mathrm{kg} \mathrm{bw} /$ day (90 day toxicity study showing mainly effects on hematological parameters) and $300 \mathrm{mg} / \mathrm{kg}$ bw/day (developmental toxicity study). ${ }^{16}$ The NOAEL of $150 \mathrm{mg} / \mathrm{kg}$ bw/day was used by EFSA as starting point to derive an ADI of $5 \mathrm{mg} / \mathrm{kg}$ bw by application of an uncertainty factor of $300 .{ }^{16}$ As far as we know, no in vivo studies are available in which thyroidhormone-related markers (T3/T4 and $\mathrm{TSH}$ ) have been measured to assess possible thyroid hormone disruption. An extended one-generation reproductive/developmental toxicity study, in which such markers are measured, is not available for propyl gallate. ${ }^{20}$ It can be expected that the in vitro observed inhibition of TPO by propyl gallate relates to its antioxidant activity. ${ }^{53}$ Inhibition of TPO is for example also observed for many flavonoids. ${ }^{62}$ To what extent the inhibition of TPO by propyl gallate actually translates into adverse effects needs to be further elucidated.

Whereas TPO inhibition by particularly propyl gallate was predicted to occur at doses that are relatively close to actual human exposure, it must be noted that in the present study, nominal effect concentrations in the bioassays are translated to oral equivalent doses based on total (bound and unbound) maximal concentrations in plasma, which can be considered as a worst-case scenario approach. Because when assuming that the free concentration of the chemical causes an adverse effect and accounting for differences between the free concentration in vitro vs in vivo, higher oral equivalent doses would be obtained, since the free fraction in an in vitro assay can in general be expected to be higher than the free fraction in plasma. Since no information on the free fraction of these chemicals in the in vitro bioassays is available, we could not refine the estimated oral equivalent doses. For assays representing intracellular targets, such as TPO in the NCCT TPO AUR dn assay, adequate estimation of oral equivalent doses is even more challenging, as the free intracellular concentrations can be considered the most relevant dose metric for QIVIVE. The result of the present study can therefore not be used for setting safe exposure levels but solely for prioritization purposes. The present study also indicates that potential effects on TPO and thyroid hormone homeostasis by propyl gallate requires further exploration.

Overall, the results of the present study support the relevance of making initial estimations of plasma and tissue concentrations of chemicals in the body with help of our webbased online tool, which allows to extrapolate in vitro effect concentrations into dose-response or potency information. The developed web-based toolbox provides a publicly available resource to perform such QIVIVE extrapolations, facilitating also nonexperienced PBK modelers to perform QIVIVE.

\section{ASSOCIATED CONTENT}

\section{Supporting Information}

The Supporting Information is available free of charge at https://pubs.acs.org/doi/10.1021/acs.chemrestox.0c00307.

Supporting Information 1: $\mathrm{R}$ codes of the human and rat PBK models applied in the online web-based toolbox (ZIP)

Supporting Information 2: LC-MS method used for chemical quantification of chemicals in in vitro incubations and workflow on how to use the webbased toolbox shown by screenshots (PDF)

\section{AUTHOR INFORMATION}

\section{Corresponding Author}

Ans Punt - Wageningen Food Safety Research, 6708 WB Wageningen, The Netherlands; (1) orcid.org/0000-00022056-4634; Phone: +31 317 481025; Email: ans.punt@ wur.nl

\section{Authors}

Nicole Pinckaers - Wageningen Food Safety Research, 6708 WB Wageningen, The Netherlands

Ad Peijnenburg - Wageningen Food Safety Research, 6708 $W B$ Wageningen, The Netherlands

Jochem Louisse - Wageningen Food Safety Research, 6708 WB Wageningen, The Netherlands

Complete contact information is available at: https://pubs.acs.org/10.1021/acs.chemrestox.0c00307

\section{Notes}

The authors declare no competing financial interest. 


\section{ACKNOWLEDGMENTS}

This work was financially supported by the Dutch Ministry of Agriculture, Nature and Food Quality (projects KB37-001-881 003 and WOT-02-002-003).

\section{REFERENCES}

(1) Louisse, J., Beekmann, K., and Rietjens, I. M. C. M. (2017) Use of Physiologically Based Kinetic Modeling-Based Reverse Dosimetry to Predict in Vivo Toxicity from in Vitro Data. Chem. Res. Toxicol. 30 (1), 114-125.

(2) Blaauboer, B. J. (2010) Biokinetic Modeling and in Vitro - in Vivo Extrapolations. J. Toxicol. Environ. Health, Part B 13 (2-4), 242252.

(3) Yoon, M., Campbell, J. L., Andersen, M. E., and Clewell, H. J. (2012) Quantitative in Vitro to in Vivo Extrapolation of Cell-Based Toxicity Assay Results. Crit. Rev. Toxicol. 42, 633.

(4) Rotroff, D. M., Wetmore, B. A., Dix, D. J., Ferguson, S. S., Clewell, H. J., Houck, K. A., LeCluyse, E. L., Andersen, M. E., Judson, R. S., Smith, C. M., Sochaski, M. A., Kavlock, R. J., Boellmann, F., Martin, M. T., Reif, D. M., Wambaugh, J. F., and Thomas, R. S. (2010) Incorporating Human Dosimetry and Exposure into HighThroughput In Vitro Toxicity Screening. Toxicol. Sci. 117 (2), 348358.

(5) COMMISSION REGULATION (EU) 2018/98 of 22 January 2018 Amending Annexes II and III to Regulation (EC) No 1333/ 2008 of the European Parliament and of the Council and the Annex to Commission Regulation (EU) No 231/2012 as Regards Calcium Sorbate (E 203). https://eur-lex.europa.eu/legal-content/EN/TXT/ ?uri=CELEX\%3A32018R0098.

(6) González, M. J., Medina, I., Maldonado, O. S., Lucas, R., and Morales, J. C. (2015) Antioxidant Activity of Alkyl Gallates and Glycosyl Alkyl Gallates in Fish Oil in Water Emulsions: Relevance of Their Surface Active Properties and of the Type of Emulsifier. Food Chem. 183, 190-196.

(7) EFSA. (2004) Opinion of the Scientific Panel on Food Additives, Flavourings, Processing Aids and Materials in Contact with Food (AFC) Related to Para Hydroxybenzoates (E 214-219). EFSA J. 2 (9), 83.

(8) Brand, W., Boon, P., Hessel, E., Meesters, J., Weda, M., and Schuur, A. Exposure to and Toxicity of Methyl-, Ethyl-and Propylparaben (2017) DOI: 10.21945/RIVM-2017-0028

(9) Hessel, E. V. S., Boon, P. E., den Braver-Sewradj, S. P., Meesters, J. A. J., Weda, M., and Brand, W. (2019) Review on Butylparaben: Exposure, Toxicity and Risk Assessment: With a Focus on Endocrine Disrupting Properties and Cumulative Risk Assessment. RIVM Report 2018-0161, National Institute for Public Health and the Environment.

(10) Golden, R., Gandy, J., and Vollmer, G. (2005) A Review of the Endocrine Activity of Parabens and Implications for Potential Risks to Human Health. Crit. Rev. Toxicol. 35, 435-458.

(11) Watanabe, Y., Kojima, H., Takeuchi, S., Uramaru, N., Ohta, S., and Kitamura, S. (2013) Comparative Study on Transcriptional Activity of 17 Parabens Mediated by Estrogen Receptor $\alpha$ and $\beta$ and Androgen Receptor. Food Chem. Toxicol. 57, 227-234.

(12) Okubo, T., Yokoyama, Y., Kano, K., and Kano, I. (2001) ERDependent Estrogenic Activity of Parabens Assessed by Proliferation of Human Breast Cancer MCF-7 Cells and Expression of ER $\alpha$ and PR. Food Chem. Toxicol. 39 (12), 1225-1232.

(13) Punt, A., Firman, J., Boobis, A., Cronin, M., Gosling, J. P., Wilks, M. F., Hepburn, P. A., Thiel, A., and Fussell, K. C. (2020) Potential of ToxCast Data in the Safety Assessment of Food Chemicals. Toxicol. Sci. 174 (2), 326-340.

(14) Amadasi, A., Mozzarelli, A., Meda, C., Maggi, A., and Cozzini, P. (2009) Identification of Xenoestrogens in Food Additives by an Integrated in Silico and in Vitro Approach. Chem. Res. Toxicol. 22 (1), $52-63$.

(15) Ter Veld, M. G. R., Schouten, B., Louisse, J., Van Es, D. S., Van Der Saag, P. T., Rietjens, I. M. C. M., and Murk, A. J. (2006)
Estrogenic Potency of Food-Packaging-Associated Plasticizers and Antioxidants as Detected in ER $\alpha$ and ER $\beta$ Reporter Gene Cell Lines. J. Agric. Food Chem. 54 (12), 4407-4416.

(16) EFSA. (2014) Scientific Opinion on the Re-evaluation of Propyl Gallate (E 310) as a Food Additive. EFSA J. 12 (4), 3642.

(17) COMMISSION REGULATION (EU) 2018/1481 of 4 October 2018 amending Annexes II and III to Regulation (EC) No 1333/2008 of the European Parliament and of the Council and the Annex to Commission Regulation (EU) No 231/2012 as regards octyl gallate (E 311) and dodecyl gallate (E 312). https://eur-lex. europa.eu/legal-content / EN / T X T / H T ML / ? uri= CELEX:32018R1481\&from=EN (accessed Jul 7, 2020).

(18) EFSA. (2015) Scientific Opinion on the Re-evaluation of Dodecyl Gallate (E 312) as a Food Additive. EFSA J. 13 (5), 4086.

(19) EFSA. (2015) Scientific Opinion on the Re-Evaluation of Octyl Gallate (E 311) as a Food Additive. EFSA J. 13 (10), 4248.

(20) Razenberg, L., and Sprong, R. Risk assessment of propyl gallate in water-based vitamin $\mathrm{D}$ supplements intended for infants and young children, RIVM letter report 2019-0217, National Institute for Public Health and the Environment. https://www.rivm.nl/bibliotheek/ rapporten/2019-0217.html (accessed Jul 7, 2020).

(21) EPA. The ToxCast Dashboard. https://actor.epa.gov/ dashboard/ (accessed Jul 7, 2020).

(22) R Core Team. R: The R Project for Statistical Computing. https://www.r-project.org/ (accessed Jul 7, 2020).

(23) RStudio. Shiny. https://rstudio.com/products/shiny/ (accessed Jul 7, 2020).

(24) Soetaert, K., Petzoldt, T., and Setzer, R. W. (2010) Solving Differential Equations in R: Package DeSolve. J. Stat. Softw. 33 (9), $1-25$.

(25) Jones, H., and Rowland-Yeo, K. (2013) Basic Concepts in Physiologically Based Pharmacokinetic Modeling in Drug Discovery and Development. CPT: Pharmacometrics Syst. Pharmacol. 2 (8), 63.

(26) Musther, H., Harwood, M. D., Yang, J., Turner, D. B., RostamiHodjegan, A., and Jamei, M. (2017) The Constraints, Construction, and Verification of a Strain-Specific Physiologically Based Pharmacokinetic Rat Model. J. Pharm. Sci. 106 (9), 2826-2838.

(27) Grandoni, S., Cesari, N., Brogin, G., Puccini, P., and Magni, P. (2019) Building In-House PBPK Modelling Tools for Oral Drug Administration from Literature Information. ADMET DMPK 7 (1), 4.

(28) Rodgers, T., and Rowland, M. (2006) Physiologically Based Pharmacokinetic Modelling 2: Predicting the Tissue Distribution of Acids, Very Weak Bases, Neutrals and Zwitterions. J. Pharm. Sci. 95 (6), $1238-1257$.

(29) Berezhkovskiy, L. M. (2004) Volume of Distribution at Steady State for a Linear Pharmacokinetic System with Peripheral Elimination. J. Pharm. Sci. 93 (6), 1628-1640.

(30) Lobell, M., and Sivarajah, V. (2003) In Silico Prediction of Aqueous Solubility, Human Plasma Protein Binding and Volume of Distribution of Compounds from Calculated PKa and AlogP98 Values. Mol. Diversity 7 (1), 69-87.

(31) Zhang, D., Luo, G., Ding, X., and Lu, C. (2012) Preclinical Experimental Models of Drug Metabolism and Disposition in Drug Discovery and Development. Acta Pharm. Sin. B 2 (6), 549-561.

(32) Barter, Z., Bayliss, M., Beaune, P., Boobis, A., Carlile, D., Edwards, R., Brian Houston, J., Lake, B., Lipscomb, J., Pelkonen, O., Tucke, G., and Rostami-Hodjegan, A. (2007) Scaling Factors for the Extrapolation of In Vivo Metabolic Drug Clearance From In Vitro Data: Reaching a Consensus on Values of Human Micro-Somal Protein and Hepatocellularity Per Gram of Liver. Curr. Drug Metab. 8 (1), 33-45.

(33) Kilford, P. J., Gertz, M., Houston, J. B., and Galetin, A. (2008) Hepatocellular Binding of Drugs: Correction for Unbound Fraction in Hepatocyte Incubations Using Microsomal Binding or Drug Lipophilicity Data. Drug Metab. Dispos. 36 (7), 1194-1197.

(34) Hallifax, D., and Houston, J. B. (2006) Binding of Drugs to Hepatic Microsomes: Comment and Assessment of Current Prediction Methodology with Recommendation for Improvement. Drug Metab. Dispos. 34 (4), 724-726. 
(35) Yu, L. X., and Amidon, G. L. (1999) A Compartmental Absorption and Transit Model for Estimating Oral Drug Absorption. Int. J. Pharm. 186 (2), 119-125.

(36) Knights, K. M., Spencer, S. M., Fallon, J. K., Chau, N., Smith, P. C., and Miners, J. O. (2016) Scaling Factors for the in Vitro - in Vivo Extrapolation (IV-IVE) of Renal Drug and Xenobiotic Glucuronidation Clearance. Br. J. Clin. Pharmacol. 81 (6), 1153-1164.

(37) Zhang, M., van Ravenzwaay, B., Fabian, E., Rietjens, I. M. C. M., and Louisse, J. (2018) Towards a Generic Physiologically Based Kinetic Model to Predict in Vivo Uterotrophic Responses in Rats by Reverse Dosimetry of in Vitro Estrogenicity Data. Arch. Toxicol. 92 (3), 1075-1088.

(38) Jones, H. M., and Houston, J. B. (2004) Substrate Depletion Approach for Determining In Vitro Metabolic Clearance: Time Dependencies in Hepatocyte and Microsomal Incubations. Drug Metab. Dispos. 32 (9), 973-982.

(39) Smith, D. A., Beaumont, K., Maurer, T. S., and Di, L. (2019) Clearance in Drug Design. J. Med. Chem. 62 (5), 2245-2255.

(40) Tsao, R. (2015) Synergistic interactions between antioxidants used in food preservation. Handbook of Antioxidants for Food Preservation, 335-347.

(41) Poulin, P., and Theil, F.-P. (2002) Prediction of Pharmacokinetics Prior to in Vivo Studies. II. Generic Physiologically Based Pharmacokinetic Models of Drug Disposition. J. Pharm. Sci. 91 (5), $1358-1370$.

(42) Hou, T. J., Zhang, W., Xia, K., Qiao, X. B., and Xu, X. J. (2004) ADME Evaluation in Drug Discovery. 5. Correlation of Caco-2 Permeation with Simple Molecular Properties. J. Chem. Inf. Comput. Sci. 44 (5), 1585-1600.

(43) Sun, D., Lennernas, H., Welage, L. S., Barnett, J. L., Landowski, C. P., Foster, D., Fleisher, D., Lee, K.-D., and Amidon, G. L. (2002) Comparison of Human Duodenum and Caco-2 Gene Expression Profiles for 12,000 Gene Sequences Tags and Correlation with Permeability of 26 Drugs. Pharm. Res. 19 (10), 1400-1416.

(44) Fagerholm, U., Johansson, M., and Lennernäs, H. (1996) Comparison between Permeability Coefficients in Rat and Human Jejunum. Pharm. Res. 13 (9), 1336-1342.

(45) Peters, S. A., and Hultin, L. (2008) Early Identification of DrugInduced Impairment of Gastric Emptying through Physiologically Based Pharmacokinetic (PBPK) Simulation of Plasma ConcentrationTime Profiles in Rat. J. Pharmacokinet. Pharmacodyn. 35 (1), 1-30.

(46) EPA. CompTox Chemicals Dashboard. https://comptox.epa. gov/dashboard (accessed Jul 20, 2020).

(47) Tullberg, S. C., Keene, W. E., Walton, K., Rakkar, P., Toor, M., and Renwick, A. G. Biomarkers, toxicokinetics and default uncertainty factors. Project Number - T01017.

(48) Aubert, N., Ameller, T., and Legrand, J. J. (2012) Systemic Exposure to Parabens: Pharmacokinetics, Tissue Distribution, Excretion Balance and Plasma Metabolites of [ 14C]-Methyl-, Propyland Butylparaben in Rats after Oral, Topical or Subcutaneous Administration. Food Chem. Toxicol. 50 (3-4), 445-454.

(49) Aubert, N., Ameller, T., and Legrand, J.-J. (2012) Systemic Exposure to Parabens: Pharmacokinetics, Tissue Distribution, Excretion Balance and Plasma Metabolites of [14C]-Methyl-, Propyland Butylparaben in Rats after Oral, Topical or Subcutaneous Administration. Food Chem. Toxicol. 50 (3-4), 445-454.

(50) Walfish, S. (2006) A Review of Statistical Outlier Methods. Pharm. Technol. 30, 1.

(51) Pendse, S. N., Efremenko, A., Hack, C. E., Moreau, M., Mallick, P., Dzierlenga, M., Nicolas, C. I., Yoon, M., Clewell, H. J., and McMullen, P. D. (2020) Population Life-Course Exposure to Health Effects Model (PLETHEM): An R Package for PBPK Modeling. Comput. Toxicol. 13, 100115.

(52) Pearce, R. G., Setzer, R. W., Strope, C. L., Sipes, N. S., and Wambaugh, J. F. (2017) Httk: R Package for High-Throughput Toxicokinetics. J. Stat. Softw. 79 (4), 1.

(53) Bossier, H., Chau, J., Ndour, C., Varewyck, M., Verbeke, T., and Vergucht, S. (2020) A Web-based Open Source Tool for
Toxicokinetic and Toxicodynamic Modelling. EFSA Support. Publ. 17 (11), 1926E.

(54) Fisher, C., Siméon, S., Jamei, M., Gardner, I., and Bois, Y. F. (2019) VIVD: Virtual in Vitro Distribution Model for the Mechanistic Prediction of Intracellular Concentrations of Chemicals in in Vitro Toxicity Assays. Toxicol. In Vitro 58, 42-50.

(55) Fischer, F. C., Henneberger, L., König, M., Bittermann, K., Linden, L., Goss, K. U., and Escher, B. I. (2017) Modeling Exposure in the Tox21 in Vitro Bioassays. Chem. Res. Toxicol. 30 (5), 11971208.

(56) Jones, H. M., Parrott, N., Jorga, K., and Lavé, T. (2006) A Novel Strategy for Physiologically Based Predictions of Human Pharmacokinetics. Clin. Pharmacokinet. 45 (5), 511-542.

(57) Peters, S. A. (2008) Evaluation of a Generic Physiologically Based Pharmacokinetic Model for Lineshape Analysis. Clin. Pharmacokinet. 47 (4), 261-275.

(58) McNally, K., Hogg, A., and Loizou, G. (2018) A Computational Workflow for Probabilistic Quantitative in Vitro to in Vivo Extrapolation. Front. Pharmacol. 9 (MAY), 508.

(59) Punt, A., et al. (2020) New Approach Methodologies (NAMs) for Human-Relevant Biokinetics Predictions. ALTEX 37, 607.

(60) Louisse, J., Alewijn, M., Peijnenburg, A. A. C. M., Cnubben, N. H. P., Heringa, M. B., Coecke, S., and Punt, A. (2020) Towards Harmonization of Test Methods for in Vitro Hepatic Clearance Studies. Toxicol. In Vitro 63, 104722.

(61) Paini, A., Leonard, J. A., Joossens, E., Bessems, J. G. M., Desalegn, A., Dorne, J. L., Gosling, J. P., Heringa, M. B., Klaric, M., Kliment, T., Kramer, N. I., Loizou, G., Louisse, J., Lumen, A., Madden, J. C., Patterson, E. A., Proença, S., Punt, A., Setzer, R. W., Suciu, N., Troutman, J., Yoon, M., Worth, A., and Tan, Y. M. (2019) Next Generation Physiologically Based Kinetic (NG-PBK) Models in Support of Regulatory Decision Making. Comput. Toxicol. 9, 61-72.

(62) Divi, R. L., and Doerge, D. R. (1996) Inhibition of Thyroid Peroxidase by Dietary Flavonoids. Chem. Res. Toxicol. 9 (1), 16-23. 